\title{
Post-Newtonian Hamiltonian description of an atom in a weak gravitational field
}

\author{
Philip K. Schwartz ${ }^{1, a}$ and Domenico Giulini ${ }^{1,2, b}$ \\ ${ }^{1}$ Institute for Theoretical Physics, Leibniz University Hannover, \\ Appelstraße 2, 30167 Hannover, Germany \\ ${ }^{2}$ Center of Applied Space Technology and Microgravity, University of Bremen, \\ Am Fallturm 1, 28359 Bremen, Germany \\ ${ }^{a}$ philip.schwartz@itp.uni-hannover.de \\ giulini@itp.uni-hannover.de
}

We extend the systematic calculation of an approximately relativistic Hamilto-
nian for centre of mass and internal dynamics of an electromagnetically bound
two-particle system by Sonnleitner and Barnett [1] to the case including
a weak post-Newtonian gravitational background field, described by the
Eddington-Robertson parametrised post-Newtonian metric. Starting from
a proper relativistic description of the situation, this approach allows to
systematically derive the coupling of the model system to gravity, instead of
'guessing' it by means of classical notions of relativistic effects.
We embed this technical result into a critical discussion concerning the
problem of implementing and interpreting general couplings to the gravita-
tional field and the connected problem of how to properly address the question
concerning the validity of the Equivalence Principle in Quantum Mechanics. 


\section{Contents}

1. Introduction 2

1.1. Logical structure of this paper . . . . . . . . . . . . . . . . . . . 5

2. A composite system in external electromagnetic and gravitational fields 6

2.1. External electromagnetic fields - the work of Sonnleitner and Barnett . . . . . . . . . 6

2.2. Including weak external gravitational fields . . . . . . . . . . . . . . . . . 7

2.3. Geometric structures, notation, and conventions . . . . . . . . . . . . . 8

3. Coupling the gravitational field to the particles $\quad \mathbf{1 0}$

3.1. The classical Hamiltonian . . . . . . . . . . . . . . . . . . . . . . . . . . . . . . 10

3.2. Canonical quantisation and PZW transformation to a multipolar Hamiltonian . . . . . 11

3.3. Introduction of centre of mass variables . . . . . . . . . . . . . . . . . . . . . 12

4. Coupling the gravitational to the electromagnetic field 13

4.1. Solution of the gravitationally modified Maxwell equations . . . . . . . . . . . . . . 13

4.2. Computation of the electromagnetic Lagrangian . . . . . . . . . . . . . . . . . . . 16

5. The total Hamiltonian including all interactions $\quad \mathbf{1 9}$

5.1. Computation of the Hamiltonian . . . . . . . . . . . . . . . . . . . . . . . . . . . 19

5.2. The system as a composite point particle . . . . . . . . . . . . . . . . 21

5.3. The electromagnetic expressions in terms of components with respect to orthonormal frames 22

6. A comment on the Equivalence Principle in Quantum Mechanics

A. Formulae from the paper of Sonnleitner and Barnett

\section{Introduction}

Experiments in quantum optics and matter-wave interferometry have now reached a degree of precision that covers 'new' relativistic corrections that were hitherto not considered in such settings. In particular, this includes couplings between 'internal' and 'centre of mass' degrees of freedom of composite systems, which have no Newtonian analogue. Hence, such experiments require proper relativistic treatments for their theoretical descriptions. Quite generally, such descriptions are often restricted to more or less ad hoc addition of 'effects' known from classical physics, like velocity-dependent masses, second-order Doppler shifts, second-order aberrations, redshifted energies, and time dilations due to relative velocities and/or gravitational potentials. Such approaches are conceptually dangerous for a number of reasons. They neither guarantee completeness and independence, nor do they need to apply in non-classical situations where quantum properties dominate the dynamics. Such 'derivations' often obscure the proper physical interpretation of relativistic corrections and, consequently, potentially misguide physical conclusions drawn form their observation.

An example of this sort has recently been discussed in the careful and lucid analysis by Sonnleitner and Barnett [1], who pointed out that certain terms that show up in computations of the interaction of moving atoms with light, and which from a non- 
relativistid ${ }^{1}$ point of view appear to be some kind of 'friction' (of a non immediately obvious physical origin, hence one may be tempted to call them 'anomalous'), are actually nothing but a straightforward consequence of Special Relativity. In fact, there is nothing to be surprised about once the calculation is done, and the results are interpreted, in a proper relativistic framework. This was done approximately in [1] for the gravity-free case, by systematically deriving an 'approximately relativistic' Hamiltonian describing the atom. It is the purpose of our paper to extend this so as to also include gravity approximately. As emphasised above, this generalisation serves not only a point of principal interest that deserves clarification, but is also of immediate practical interest, not only in the obvious realm of quantum optics experiments regarding the detection of gravitational waves, but also in atom interferometry; see, e.g. [2] 8$]$. A calculation using methods very similar to those of [1] including external gravitational fields was performed by Marzlin already in 1995 [9] $]^{2}$ but unlike Sonnleitner and Barnett in [1] or our calculation in this work, Marzlin did not perform a full first-order post-Newtonian expansion and instead focused on the electric dipole coupling only.

What is generally needed is a consistent post-Newtonian approximation scheme in which the transition from Galilei invariant to Poincaré invariant (Special Relativity) and further to diffeomorphism invariant (General Relativity) laws can be systematically derived in a step-by-step algorithmic fashion. Such a scheme must be fundamentally rooted in a principle that contains all the information of how matter couples to gravity.

For classical matter, described in terms of dynamical laws compatible with the requirements of Special Relativity, such a principle is known in form of the 'minimal coupling scheme'. According to this scheme, the Minkowski metric of Special Relativity is to be replaced by the more general Lorentzian metric of spacetime and all partial derivatives (or more precisely: Levi-Civita covariant derivatives with respect to the Minkowski metric) are to be replaced with the Levi-Civita covariant derivatives with respect to the general spacetime metric. This scheme is based on Einstein's Equivalence Principle, whose core statement is that gravity can be encoded in the geometry of spacetime that is common to all matter components. We stress that this is the important point encoding the universality of gravitational interaction: that any matter component, may it be light, neutrinos, or other elementary particles with or without mass, spin, charge, or other features, or may it be a macroscopic body, like a football or a planet, all of them will couple to gravity in a way that only depends on one and the same geometry of spacetime; compare [10].

The problem we face in quantum physics as regards its coupling to gravitational fields is that the minimal coupling scheme has no straightforward generalisation to Galilei invariant

\footnotetext{
${ }^{1}$ As a matter of principle, we would like to avoid the common but misleading adjective 'non-relativistic' to distinguish Galilei invariant dynamical laws from 'relativistic' ones, by which one then means those obeying Poincaré invariance. It is not the validity of the physical relativity principle that distinguishes both cases. Rather, their difference lies in the way that principle is implemented. Since we cannot entirely escape traditionally established nomenclature without undue complications in expression, we will continue to use the term 'non-relativistic' in the sense just explained and think of it as always being put between (invisible) inverted commas.

${ }^{2}$ We are grateful to Alexander Friedrich for pointing out this reference to us.
} 
Quantum Mechanics, for the obvious reason that the latter is incompatible with the requirements of Special Relativity. As is well known, enforcing Poincaré symmetry upon Quantum Mechanics eventually leads to the framework of Poincaré invariant Quantum Field Theory, often referred to as 'Relativistic Quantum Field Theory' (RQFT), whose mathematical structure and physical interpretation is far more complex than that of ordinary non-relativistic Quantum Mechanics. As a matter of principle, a post-Newtonian expansion should therefore start from RQFT.

However, if one is merely interested in leading order 'relativistic corrections' below the threshold of pair production for any of the massive particles involved, a simpler method is to first put the classical system in a fixed particle sector into Poincare invariant form, then apply the minimal coupling scheme on the classical level, and finally apply suitable rules for quantisation, like the canonical ones. This is the procedure we will follow in this paper. It is computationally and conceptually much cheaper than a proper quantum field theoretic treatment and is a priori limited to sectors of fixed numbers in each massive particle species (hence it would not make sense to carry the computation of 'relativistic corrections' further than to the lowest lying particle-production threshold). In fact, the corrections we are after are far from those thresholds and may be safely derived from a systematic post-Newtonian expansion on the classical level.

It is the underlying systematics of producing 'relativistic corrections' that, in our opinion, distinguishes our approach from others, like, e.g., [3 8]. These latter attempts make use in an essential way of notions, like 'wordline' and 'redshift', which have no immediate meaning in quantum theory, unless the state of the system is severely restricted in an a priori fashion. More precisely, the state of the total system (the atom) is assumed to unambiguously define a worldline, say for the centre of mass, which can also be assigned a length that is then identified with the proper time (up to division by a factor of $c$ ). This implies that 1) the overall pure state of the system separates into the tensor product of a pure state for the centre of mass with a pure state for the relative degrees of freedom, and that 2) the state for the centre of mass is of semiclassical nature, so as to unambiguously determine a piecewise smooth $\left(C^{1}\right)$ worldline. ${ }^{3}$ It may well be that these a priori restrictions can be justified in specific applications within quantum optics and atom interferometry. However, here we wish to promote the view that the theoretical problem of formulating a consistent post-Newtonian coupling scheme should be solved independently of such restrictions.

In addition, phrasing relativistic corrections in terms of classical notions like 'proper times', 'redshifts', and the like bears the danger of losing control over aspects of completeness and possible redundancies. To be sure, these classical 'effects' will appear as consequences from the general scheme, if applied to the specific situation that allows such vocabulary. But they should not be mistaken for the relativistic corrections proper, which derive from the spacetime geometry as a whole that enters the quantum dynamical laws at a fundamental level. Similar remarks apply in connection with attempts to

\footnotetext{
${ }^{3}$ We recall that the path integral in ordinary Quantum Mechanics generally receives contributions from continuous but nowhere differentiable paths. Only in very special situations is the dominant contribution given by the action along a smooth classical path.
} 
formulate the Equivalence Principle in Quantum Mechanics. Since we consider this to be an important and directly related issue, which also bears the danger of misconceptions, we will devote an extra section to its discussion at the end of this paper.

Problems of this sort were avoided by Sonnleitner and Barnett [1] by the means of basing their whole calculation on a proper relativistic treatment of the situation (an atom interacting with an external electromagnetic field). In the end, the first-order post-Newtonian Hamiltonian they obtained could then be used to interpret aspects of the situation in terms of classical 'relativistic corrections': The 'centre of mass' part of the final Hamiltonian has the form of a single-particle kinetic Hamiltonian, where the rôle of the rest-mass of this particle is played by the total mass-energy of the atom, i.e. the sum of the rest masses of the consituent particles and the internal atomic energy divided by $c^{2}$. Thus, the computation in [1] explicitly shows that this physically intuitive picture of a 'composite particle', suggested by mass-energy equivalence, can, in fact, be derived in a controlled and systematic approximation scheme, rather than merely made plausible from semi-intuitive physical considerations.

As will be shown in this paper, a similar interpretation is possible for the situation including external gravitational fields: When expressing the final Hamiltonian using the physical spacetime metric, an intuitive 'composite point particle' picture including the 'mass defect' due to mass-energy equivalence will again be available for the centre of mass dynamics. Our essential result in that respect is expressed by equations (5.5) to (5.8). This lends justification based on detailed calculations within systematic approximation schemes to some of the naiver approaches that are based on a priori assumptions concerning the gravity-matter coupling.

\subsection{Logical structure of this paper}

In section 2, we set up the background for our calculations: after describing the physical system under consideration, we will give an overview over the method of computation in [1]. Then we will discuss the geometric structures necessary to perform a post-Newtonian limit and to describe weak gravitational fields.

In the following, we will compute in detail the 'gravitational corrections' to the calculation by Sonnleitner and Barnett [1] arising from the presence of the gravitational field. Section 3 will deal with the coupling of the gravitational field to the kinetic terms of the particles only, ignoring couplings of the gravitational to the electromagnetic field.

In section 4, we will then compute the Lagrangian of the electromagnetic field in the presence of the gravitational field. This allows us to compute the total Hamiltonian describing the atomic system in section 5.1 , by repeating the calculation from section 3 while including the 'gravitational corrections' to electromagnetism obtained in section 4 . The resulting Hamiltonian will then be interpreted in terms of the physical spacetime metric and compared to earlier results in the remainder of section 5 .

In section 6, we will critically discuss attempts of formulating the Equivalence Principle in Quantum Mechanics, before concluding in section 7 with a brief summary of our paper. In sections 3 and 5.1 we will very closely follow the calculation from and presentation in [1]. For the reader's convenience, we have reproduced all formulae from [1] that are 
used in our calculation in the appendix. We use the original numbering, prepended with '[1].', so for example ([1.25f) refers to equation (25f) of [1]. As some of the equations from [1] contain minor errors (mostly sign errors), we give the corrected versions in the

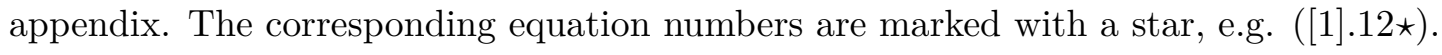

\section{A composite system in external electromagnetic and gravitational fields}

We consider a simple system consisting of two particles without spin, with respective electric charges $e_{1}, e_{2}$, masses $m_{1}, m_{2}$, and spatial positions $\mathbf{r}_{1}, \mathbf{r}_{2}$. For simplicity we assume the charges to be equal and opposite, i.e., $e_{2}=-e_{1}=: e$. In what follows, we will take into account their mutual electromagnetic interaction but neglect their mutual gravitational interaction. This two-particle system, which we will sometimes refer to as 'atom', will be placed in an external electromagnetic field, which we will take into account, as well as an external gravitational field, that we will also take into account. It is the inclusion of the latter that we wish to discuss in this paper and that extends previous studies [1].

\subsection{External electromagnetic fields - the work of Sonnleitner and Barnett}

In [1], Sonnleitner and Barnett describe a systematic method to obtain an 'approximately relativistic' quantum Hamiltonian for a system as described above interacting with an external electromagnetic field, where 'approximately relativistic' refers to the inclusion of lowest order post-Newtonian correction terms, i.e. of order $c^{-2}$. Their work was motivated by their own observation [11, 12] that the electromagnetic interaction of a decaying atom, which in QED follows an intrinsically special-relativistic symmetry (i.e. Poincaré invariance), will give rise to unnaturally looking friction-like terms that seem to contradict the relativity principle (which, of course, they don't) if interpreted in a non-relativistic (i.e. Galilei invariant) setting of ordinary Quantum Mechanics. Their correct conclusion in [1] was that this confusion can be altogether avoided by replacing this 'hotchpotch' (their wording, see last line on p. 042106-9 of [1]) of symmetry concepts by a systematic post-Newtonian derivation starting from a common, manifestly Poincaré symmetric description.

As our development will closely follow theirs, let us describe the strategy of [1] in more detail. They start with the classical Poincaré invariant Lagrangian function describing the situation, where the kinetic terms of the two particles are expanded to post-Newtonian order. This Lagrangian includes the electromagnetic fields generated by the particles themselves as lowest-order solutions of the Maxwell equations, thus eliminating the 'internal' field degrees of freedom.

This classical Lagrangian (the sum of the famous Darwin Lagrangian [13] and the external field Lagrangian) is then Legendre transformed and canonically quantised to obtain a quantum Hamiltonian in what they call the 'minimal coupling form'. They then perform a Power-Zienau-Woolley (PZW) unitary transformation [14-16] together with a 
multipolar expansion of the external field in order to transform the Hamiltonian into a so-called 'multipolar form'.

Then, introducing Newtonian centre of mass and relative coordinates $\mathbf{R}, \mathbf{r}$, and the corresponding canonical momenta $\mathbf{P}, \mathbf{p}_{\mathbf{r}}$, they arrive at what they call the centre of mass Hamiltonian. This can be separated into parts describing the central motion of the atom, the internal atomic motion, the external electromagnetic field, and its interaction with the atom. However, this Hamiltonian also contains cross terms coupling the relative degrees of freedom to the central momentum $\mathbf{P}$. In order to eliminate this coupling, they perform a final canonical transformation to new coordinates $\mathbf{Q}, \mathbf{q}$ and momenta $\mathbf{P}, \mathbf{p}$.

\subsection{Including weak external gravitational fields}

As already stated above, our contribution in this paper will consist in generalising the calculation of [1] to the case of the atom being situated in a weak external gravitational field in addition to the electromagnetic field already considered in [1]. Our aim is to likewise obtain an 'approximately relativistic', i.e. first-order post-Newtonian, Hamiltonian describing this situation.

Conceptually speaking, this generalisation is not entirely obvious for the following reason: The addition of gravitational fields will, according to General Relativity, result in a changed geometry of spacetime and, consequently, in the loss of some or all spacetime symmetries and their associated conservation laws. In particular, Poincaré symmetry will be lost and there is no obvious way to implement the post-Newtonian expansion employed in [1]. In fact, the concept of a 'post-Newtonian expansion' in an arbitrary spacetime simply does not exist without the explicit introduction of certain background structures that give meaning to notions like 'weak' gravitational fields and 'slow' velocities. To cut a long story short, such necessary background structures will in our case be 1) the Poincaré symmetric Minkowski metric $\eta$ on spacetime $M$ and 2) a preferred inertial reference frame in Minkowski space $(M, \eta)$, mathematically represented by a timelike vector field $u$ which is geodesic for $\eta$.

That the gravitational field be weak then means that the physical spacetime metric $g$ deviates only little from the Minkowski metric $\eta$. More precisely this means in our case that quadratic and higher orders of the difference $h:=g-\eta$ and its derivatives can be neglected. That velocities be slow means that the velocity $v$ of each particle relative to the preferred frame is small to $c$, so that terms in $v / c$ of higher order than the second can be neglected.

We stress that all the structures introduced and all the conditions of 'weakness' and 'slowness' mentioned are totally independent of coordinates that we may choose to parametrise spacetime. That is not to say that there may not be preferred coordinates which are particularly adapted to the given background structure. Indeed, such adapted coordinates obviously exist, namely so-called inertial coordinates $\left\{x^{0}, x^{1}, x^{2}, x^{3}\right\}$ in Minkowski space $(M, \eta)$, such that $x^{0}=c t, u=\partial / \partial t$, and $\eta=\eta_{\mu \nu} \mathrm{d} x^{\mu} \otimes \mathrm{d} x^{\nu}$ with $\left(\eta_{\mu \nu}\right)=\operatorname{diag}(-1,1,1,1)$. In the same coordinate system, the components of the physical 
spacetime metric $g=g_{\mu \nu} \mathrm{d} x^{\mu} \otimes \mathrm{d} x^{\nu}$ that we consider here are then given by

$$
\left(g_{\mu \nu}\right)=\left(\begin{array}{cc}
-1-2 \frac{\phi}{c^{2}}-2 \beta \frac{\phi^{2}}{c^{4}}+\mathrm{O}\left(c^{-6}\right) & \mathrm{O}\left(c^{-5}\right) \\
\mathrm{O}\left(c^{-5}\right) & \left(1-2 \gamma \frac{\phi}{c^{2}}\right) \mathbb{1}+\mathrm{O}\left(c^{-4}\right)
\end{array}\right)
$$

where $\phi$ is a scalar function on spacetime that may be seen as the analogue of the Newtonian gravitational potential in this approximation scheme. The weakness of the gravitational field is expressed in components by $\left|h_{\mu \nu}\right|=\left|g_{\mu \nu}-\eta_{\mu \nu}\right| \ll 1$. Using (2.1) this is equivalent to the smallness of the Newtonian potential as compared to $c^{2}$, that is $\phi / c^{2} \ll 1$.

The metric (2.1) also contains two dimensionless parameters $\beta$ and $\gamma$, the so-called 'Eddington-Robertson parameters', which we introduced in order to account for possible deviations from General Relativity. General Relativity corresponds to the values $\beta=$ $\gamma=1$, in which case the metric (2.1) solves the field equations of General Relativity approximately in a $1 / c$-expansion for a static source, with $\phi$ being the Newtonian gravitational potential of the source. The metrics for different values of these parameters are then considered to correspond to so-called 'test theories' against which the predictions of General Relativity can be tested; see, e.g., [17]. Following standard terminology, we shall refer to (2.1) as the 'PPN metric' (parametrised post-Newtonian). The explicit inclusion of $\beta$ and $\gamma$ in our formalism allows us to track the consequences of postNewtonian corrections in the spatial and the temporal part of the metric separately. This also opens the possibility to apply our results to potential future quantum tests of General Relativity itself, which are outside the scope of this paper.

For later use, we introduce the 'physical spatial metric' ${ }^{(3)} g$, which is the restriction of the physical spacetime metric $g$ to three-dimensional 'space', i.e. to the orthogonal complement of the preferred vector field $u$. The inverse of this physical spatial metric will be denoted by ${ }^{(3)} g^{-1}$.

Later we will also need the inverse metric to $g$, the components of which in the specified coordinate system are simply obtained by inverting the matrix (2.1):

$$
\left(g^{\mu \nu}\right)=\left(\begin{array}{cc}
-1+2 \frac{\phi}{c^{2}}+(2 \beta-4) \frac{\phi^{2}}{c^{4}}+\mathrm{O}\left(c^{-6}\right) & \mathrm{O}\left(c^{-5}\right) \\
\mathrm{O}\left(c^{-5}\right) & \left(1+2 \gamma \frac{\phi}{c^{2}}\right) \mathbb{1}+\mathrm{O}\left(c^{-4}\right)
\end{array}\right)
$$

Since we are interested in a lowest-order post-Newtonian description, we will work up to (and including) terms of order $c^{-2}$ and neglect higher order terms. In fact, corrections of higher order cannot be treated in a simple Hamiltonian formalism as employed here, without explicitly including the internal electromagnetic field degrees of freedom as dynamical variables: Elimination of the internal field variables by solving Maxwell's equations will introduce retardation effects at higher orders, thus leading to an action that is non-local in time, spoiling the application of the Hamiltonian formalism.

\subsection{Geometric structures, notation, and conventions}

The chosen background structures that allow us to define the approximation scheme (according to 'slow' and 'weak') are also employed in developing our calculation in parallel 
with that in [1]. More precisely, we use the background Minkowski metric $\eta$ and the preferred timelike vector field $u$ to decompose spacetime into time (integral lines of $u$ ) and space (hyperplanes $\eta$-perpendicular to $u$ ), and to endow space with a flat Riemannian metric (the restriction of $\eta$ to the hyperplanes). With that structure 'space' just becomes ordinary flat Euclidean space. We are indeed free to use this 'flat' structure to perform all our computations, the benefit being the aimed-for direct comparison with [1]. However, once the results of the computations are established, we have to keep in mind that physical distances and times are measured with the physical metric $g$, not the auxiliary metric $\eta$. We will see that it is precisely this re-interpretation of the formulae obtained that lends them good physical meaning.

In our calculations, vectors and tensors will be represented by their components with respect to the chosen coordinate system $\left(x^{\mu}\right)=\left(c t, x^{a}\right)$. We let greek indices run from 0 to 3 , latin indices from 1 to 3 and we shall use the Einstein summation convention for like indices at different levels (one up- one downstairs). Indices are lowered and raised by the physical spacetime metric $g_{\mu \nu}$ and its inverse $g^{\mu \nu}$ respectively. The Minkowski metric takes its usual diagonal form (as stated above) and the spatial metric its usual Euclidean form with components of the metric tensor given by $\left(\delta_{a b}\right)=\operatorname{diag}(1,1,1)$ and its inverse $\left(\delta^{a b}\right)=\operatorname{diag}(1,1,1)$.

We will often employ a 'three-vector' notation, where the three-tuple of spatial components of some quantity will be denoted by an upright, boldface letter: for example, $\mathbf{r}_{1}$ is the 'vector' of spatial coordinates of the first particle. When using this notation, a dot between two such 'vectors' will denote the component-wise 'Euclidean scalar product', i.e.

$$
\mathbf{v} \cdot \mathbf{w}:=\delta_{a b} v^{a} w^{b}=\sum_{a=1}^{3} v^{a} w^{a},
$$

and a cross multiplication symbol will denote the component-wise vector product, i.e.

$$
(\mathbf{v} \times \mathbf{w})^{a}:=\delta^{a n} \varepsilon_{n b c} v^{b} w^{c}
$$

where $\varepsilon_{a b c}$ is the usual totally antisymmetric symbol. Geometrically, $\varepsilon_{a b c}$ can be understood as the components of the spatial volume form induced by the Euclidean metric. We will lower and raise the indices of $\varepsilon$ by $\delta_{a b}$ and $\delta^{a b}$ respectively, i.e. $\varepsilon_{b c}^{a}:=\delta^{a n} \varepsilon_{n b c}$ etc., such that we can write $(\mathbf{v} \times \mathbf{w})^{a}=\varepsilon^{a}{ }_{b c} v^{b} w^{c}$.

A boldface nabla symbol $\boldsymbol{\nabla}$ denotes the three-tuple of partial derivatives,

$$
\boldsymbol{\nabla}=\left(\partial_{1}, \partial_{2}, \partial_{3}\right),
$$

which can be geometrically understood as the component representation of the spatial covariant derivatives with respect to the flat euclidean metric. It will be used to express component-wise vector calculus operations in the usual short-hand notation, for example writing

$$
(\boldsymbol{\nabla} \times \mathbf{A})^{a}=\varepsilon^{a b c} \partial_{b} A_{c}
$$

for the component-wise curl of $\mathbf{A}$. 
In view of the structures introduced we stress again that all the operations reported here and used in the sequel make good geometric sense. They do depend on the geometric structures that we made explicit above, but they do not depend on the coordinates or frames that one uses in order to express the geometric objects (including the background structures) in terms of their real-valued components. This fact is very important to keep in mind if it comes to the task of interpreting the results of computations. For example, these results will contain geometric operations, like scalar products, which my be taken using either of the metric structures provided by the formalism. What may then appear as a more or less complicated gravitational correction to the flat space result will then, in fact, turn out to be a simple and straightforward transcription of the latter into the proper physical metric, as one might have anticipated from some more or less naive working-version of the Equivalence Principle. Interpretational issues like this are well known in the literature on gravitational couplings of quantum systems; see, e.g., [9, 18]. For us, too, they will once more turn out to be relevant in connection with the total Hamiltonian in section 5.1 and the ensuing discussion in section 5.3 . We will derive and interpret the relevant gravitational terms relative to the background structures $(\eta, u)$ in order to keep the analogy with the computation in [1, but then we shall re-interpret the results in terms of the proper physical metric $g$ in order to reveal their naturalness.

\section{Coupling the gravitational field to the particles}

In this section we will work out the influence of the gravitational field when coupled to the kinetic terms of the particles only, ignoring its couplings to the electromagnetic field. The latter will be the subject of the following sections.

Starting from the Lagrangian for our atom in the absence of gravity and adding the 'gravitational corrections' to the kinetic terms of the particles, we will then repeat the calculation of [1] to obtain a quantum Hamiltonian in centre of mass coordinates.

\subsection{The classical Hamiltonian}

For a single free point particle with mass $m$ and position $\mathbf{x}$, the classical kinetic Lagrangian (parametrising the worldline by coordinate time) in our metric 2.1 reads

$$
\begin{aligned}
L_{\text {point }} & =-m c^{2} \sqrt{-g_{\mu \nu} \dot{x}^{\mu} \dot{x}^{\nu} / c^{2}} \\
& =\frac{m \dot{\mathbf{x}}^{2}}{2}\left(1+\frac{\dot{\mathbf{x}}^{2}}{4 c^{2}}\right)-m c^{2}-m \phi\left(1+(2 \beta-1) \frac{\phi}{2 c^{2}}\right)-\frac{2 \gamma+1}{2} \frac{m \phi}{c^{2}} \dot{\mathbf{x}}^{2}+\mathrm{O}\left(c^{-4}\right) .
\end{aligned}
$$

Now considering our two-particle system, the kinetic terms for the particles in gravity are given as the sum of two terms as in (3.1). These lowest-order 'gravitationally corrected' kinetic terms we include into the classical Lagrangian from $[1] .4$ which described two particles interacting with an electromagnetic field in the absence of gravity.

\footnotetext{
${ }^{4}$ We remind the reader that all the equations from [1] that we refer to explicitly are reproduced in the appendix.
} 
Eliminating the internal electromagnetic fields literally as in [1], we arrive at the post-Newtonian classical Lagrangian

$$
\begin{aligned}
L_{\text {new }}= & L-m_{1} \phi\left(\mathbf{r}_{1}\right)-m_{2} \phi\left(\mathbf{r}_{2}\right)-\frac{2 \gamma+1}{2} \frac{m_{1} \phi\left(\mathbf{r}_{1}\right)}{c^{2}} \dot{\mathbf{r}}_{1}^{2}-\frac{2 \gamma+1}{2} \frac{m_{2} \phi\left(\mathbf{r}_{2}\right)}{c^{2}} \dot{\mathbf{r}}_{2}^{2} \\
& -(2 \beta-1) \frac{m_{1} \phi\left(\mathbf{r}_{1}\right)^{2}}{2 c^{2}}-(2 \beta-1) \frac{m_{2} \phi\left(\mathbf{r}_{2}\right)^{2}}{2 c^{2}}
\end{aligned}
$$

describing our electromagnetically bound two-particle system and the external electromagnetic field. Here $L$ is the final classical Lagrangian from (1].8), (1].9).

Legendre transforming this with respect to the particle velocities $\dot{\mathbf{r}}_{i}$ and the time derivative $\partial_{t} \mathbf{A}^{\perp}$ of the electromagnetic vector potential, we obtain the total classical Hamiltonian

$$
\begin{aligned}
H_{\text {new }}= & H+m_{1} \phi\left(\mathbf{r}_{1}\right)+m_{2} \phi\left(\mathbf{r}_{2}\right)+\frac{2 \gamma+1}{2 m_{1} c^{2}} \phi\left(\mathbf{r}_{1}\right) \overline{\mathbf{p}}_{1}^{2}+\frac{2 \gamma+1}{2 m_{2} c^{2}} \phi\left(\mathbf{r}_{2}\right) \overline{\mathbf{p}}_{2}^{2} \\
& +(2 \beta-1) \frac{m_{1} \phi\left(\mathbf{r}_{1}\right)^{2}}{2 c^{2}}+(2 \beta-1) \frac{m_{2} \phi\left(\mathbf{r}_{2}\right)^{2}}{2 c^{2}}
\end{aligned}
$$

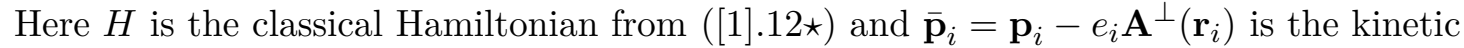
momentum. Note that we dropped all terms that go beyond our order of approximation.

\subsection{Canonical quantisation and PZW transformation to a multipolar Hamiltonian}

Now, we canonically quantise this Hamiltonian and perform the PZW transformation and electric dipole approximation used in [1] to arrive at the 'multipolar' Hamiltonian from $\left[1.23 \star\right.$. Neglecting terms of the form $\frac{\mathbf{p}_{i} \cdot[\mathbf{d} \times \mathbf{B}(\mathbf{R})]}{m_{i} m_{j} c^{2}}$ as in $[1.22$, in our gravitational correction terms from (3.3) these transformations amount just to the replacement $\overline{\mathbf{p}}_{i} \rightarrow \mathbf{p}_{i}$ (compare the appendix from $([1] .14 \star$ to $(1] .21 \star)$ ). Hence the multipolar Hamiltonian including the gravitational correction terms is

$$
\begin{aligned}
H_{[\text {mult }] \text {,new }}= & H_{[\text {mult }]}+m_{1} \phi\left(\mathbf{r}_{1}\right)+m_{2} \phi\left(\mathbf{r}_{2}\right)+\frac{2 \gamma+1}{2 m_{1} c^{2}} \mathbf{p}_{1} \cdot \phi\left(\mathbf{r}_{1}\right) \mathbf{p}_{1}+\frac{2 \gamma+1}{2 m_{2} c^{2}} \mathbf{p}_{2} \cdot \phi\left(\mathbf{r}_{2}\right) \mathbf{p}_{2} \\
& +(2 \beta-1) \frac{m_{1} \phi\left(\mathbf{r}_{1}\right)^{2}}{2 c^{2}}+(2 \beta-1) \frac{m_{2} \phi\left(\mathbf{r}_{2}\right)^{2}}{2 c^{2}}
\end{aligned}
$$

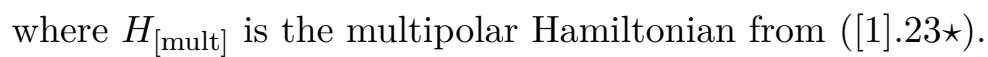

Now that we are on the quantum level, we had to choose a symmetrised operator ordering for the $\mathbf{p}^{2} \phi$ terms. We chose an ordering of the form $\mathbf{p} \cdot \phi \mathbf{p}$ since that is an 'obvious' choice, which also results if a WKB-like expansion of the Klein-Gordon equation is used for the description of single quantum particles in an Eddington-Robertson PPN metric [19], when one neglects terms involving $\Delta \phi$ (which vanishes outside the matter generating the Newtonian potential $\phi$ ). 


\subsection{Introduction of centre of mass variables}

We now want to express the correction terms in (Newtonian) centre of mass and relative variables,

$$
\begin{aligned}
\mathbf{R} & =\frac{m_{1} \mathbf{r}_{1}+m_{2} \mathbf{r}_{2}}{M}, & \mathbf{r} & =\mathbf{r}_{1}-\mathbf{r}_{2}, \\
\mathbf{P} & =\mathbf{p}_{1}+\mathbf{p}_{2}, & \mathbf{p}_{1,2} & =\frac{m_{1,2}}{M} \mathbf{P} \pm \mathbf{p}_{\mathbf{r}}
\end{aligned}
$$

where $M=m_{1}+m_{2}$. To this end, we expand the gravitational potential $\phi$ around the centre of mass position $\mathbf{R}$ in linear order (i.e. perform a monopole approximation of the generating mass distribution). In this approximation, we have $m_{1} \phi\left(\mathbf{r}_{1}\right)+m_{2} \phi\left(\mathbf{r}_{2}\right)=$ $M \phi(\mathbf{R})$ and $m_{1} \phi\left(\mathbf{r}_{1}\right)^{2}+m_{2} \phi\left(\mathbf{r}_{2}\right)^{2}=M \phi(\mathbf{R})^{2}$. Furthermore using

$$
\begin{aligned}
\mathbf{p}_{1,2} \cdot \phi\left(\mathbf{r}_{1,2}\right) \mathbf{p}_{1,2} & =\left(\frac{m_{1,2}}{M} \mathbf{P} \pm \mathbf{p}_{\mathbf{r}}\right) \cdot \phi\left(\mathbf{r}_{1,2}\right)\left(\frac{m_{1,2}}{M} \mathbf{P} \pm \mathbf{p}_{\mathbf{r}}\right) \\
& =\frac{m_{1,2}^{2}}{M^{2}} \mathbf{P} \cdot \phi\left(\mathbf{r}_{1,2}\right) \mathbf{P} \pm \frac{m_{1,2}}{M}\left(\mathbf{P} \cdot \phi\left(\mathbf{r}_{1,2}\right) \mathbf{p}_{\mathbf{r}}+\text { H.c. }\right)+\mathbf{p}_{\mathbf{r}} \cdot \phi\left(\mathbf{r}_{1,2}\right) \mathbf{p}_{\mathbf{r}}
\end{aligned}
$$

and the relations $\phi\left(\mathbf{r}_{1}\right)-\phi\left(\mathbf{r}_{2}\right)=\mathbf{r} \cdot \boldsymbol{\nabla} \phi(\mathbf{R})$ as well as

$$
\begin{aligned}
\frac{1}{m_{1}} \phi\left(\mathbf{r}_{1}\right)+\frac{1}{m_{2}} \phi\left(\mathbf{r}_{2}\right) & =\left(\frac{1}{m_{1}}+\frac{1}{m_{2}}\right) \phi(\mathbf{R})+\frac{1}{M}\left(\frac{m_{2}}{m_{1}}-\frac{m_{1}}{m_{2}}\right) \mathbf{r} \cdot \nabla \phi(\mathbf{R}) \\
& =\frac{1}{\mu} \phi(\mathbf{R})-\frac{m_{1}-m_{2}}{m_{1} m_{2}} \mathbf{r} \cdot \boldsymbol{\nabla} \phi(\mathbf{R})
\end{aligned}
$$

where $\mu=\frac{m_{1} m_{2}}{M}$ is the system's reduced mass, we arrive at the centre of mass Hamiltonian

$$
\begin{aligned}
H_{[\mathrm{com}], \text { new }}= & H_{[\mathrm{com}]}+M \phi(\mathbf{R})+(2 \beta-1) \frac{M \phi(\mathbf{R})^{2}}{2 c^{2}}+\frac{2 \gamma+1}{2 M c^{2}} \mathbf{P} \cdot \phi(\mathbf{R}) \mathbf{P} \\
& +\frac{2 \gamma+1}{2 \mu c^{2}} \mathbf{p}_{\mathbf{r}}^{2} \phi(\mathbf{R})+\frac{2 \gamma+1}{2 M c^{2}}\left[\mathbf{P} \cdot(\mathbf{r} \cdot \nabla \phi(\mathbf{R})) \mathbf{p}_{\mathbf{r}}+\text { H.c. }\right] \\
& -\frac{2 \gamma+1}{2 c^{2}} \frac{m_{1}-m_{2}}{m_{1} m_{2}} \mathbf{p}_{\mathbf{r}} \cdot(\mathbf{r} \cdot \nabla \phi(\mathbf{R})) \mathbf{p}_{\mathbf{r}},
\end{aligned}
$$

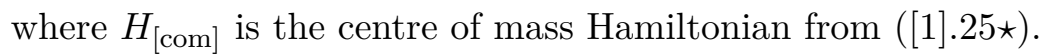

This can, as in [1], be brought into the form

$$
H_{\text {[com],new }}=H_{\mathrm{C}, \text { new }}+H_{\mathrm{A}, \text { new }}+H_{\mathrm{AL}}+H_{\mathrm{L}}+H_{\mathrm{X}, \text { new }}
$$

where

$$
H_{\mathrm{C}, \text { new }}=H_{\mathrm{C}}+\frac{2 \gamma+1}{2 M c^{2}} \mathbf{P} \cdot \phi(\mathbf{R}) \mathbf{P}+\left(M+\frac{\mathbf{p}_{\mathbf{r}}^{2}}{2 \mu c^{2}}\right) \phi(\mathbf{R})+(2 \beta-1) \frac{M \phi(\mathbf{R})^{2}}{2 c^{2}}
$$

describes the dynamics of the centre of mass and

$$
H_{\mathrm{A}, \text { new }}=H_{\mathrm{A}}+2 \gamma \frac{\phi(\mathbf{R})}{c^{2}} \frac{\mathbf{p}_{\mathbf{r}}^{2}}{2 \mu}-\frac{2 \gamma+1}{2 c^{2}} \frac{m_{1}-m_{2}}{m_{1} m_{2}} \mathbf{p}_{\mathbf{r}} \cdot(\mathbf{r} \cdot \nabla \phi(\mathbf{R})) \mathbf{p}_{\mathbf{r}}
$$


describes the internal dynamics of the atom, both modified in comparison to [1]. Here, we have included the term $2 \gamma \frac{\phi(\mathbf{R})}{c^{2}} \frac{\mathbf{p}_{\mathbf{r}}^{2}}{2 \mu}$ into $H_{\mathrm{A} \text {,new }}$ since it can be combined with $\frac{\mathbf{p}_{\mathbf{r}}^{2}}{2 \mu}$ from $H_{\mathrm{A}}$ into

$$
\frac{\mathbf{p}_{\mathbf{r}}^{2}}{2 \mu}\left(1+2 \gamma \frac{\phi(\mathbf{R})}{c^{2}}\right)=\frac{{ }^{(3)} g_{\mathbf{R}}^{-1}\left(\mathbf{p}_{\mathbf{r}}, \mathbf{p}_{\mathbf{r}}\right)}{2 \mu}
$$

giving the geometrically correctly defined Newtonian internal kinetic energy, using the metric square of the internal momentum. Here ${ }^{(3)} g_{\mathbf{R}}^{-1}$ denotes the inverse of the physical spatial metric at position $\mathbf{R}$, as explained before 2.2 .

The terms $H_{\mathrm{AL}}$ and $H_{\mathrm{L}}$ describing, respectively, the atom-light interaction and the electromagnetic field are not changed compared to [1]; and the final summand

$$
H_{\mathrm{X}, \text { new }}=H_{\mathrm{X}}+\frac{2 \gamma+1}{2 M c^{2}}\left[\mathbf{P} \cdot(\mathbf{r} \cdot \boldsymbol{\nabla} \phi(\mathbf{R})) \mathbf{p}_{\mathbf{r}}+\text { H.c. }\right]
$$

containing 'cross terms' coupling the internal degrees of freedom to the central momentum $\mathbf{P}$ gains an additional term.

Note that if we assume that the gravitational potential $\phi$ vary slowly over the extension of the atom, we can neglect the terms $\mathbf{r} \cdot \boldsymbol{\nabla} \phi(\mathbf{R})$.

\section{Coupling the gravitational to the electromagnetic field}

Having determined the gravitational field's coupling to the particles in the previous section, we now turn to its coupling to the electromagnetic field. In the following section 5 we will then combine all couplings into a single Hamiltonian.

\subsection{Solution of the gravitationally modified Maxwell equations}

The electromagnetic part of the action, including interaction with matter, is

$$
S_{\mathrm{em}}=\int \mathrm{d} t \mathrm{~d}^{3} \mathbf{x} \sqrt{-g}\left(-\frac{1}{4 \mu_{0}} F_{\text {tot. } \mu \nu} F_{\text {tot. }}^{\mu \nu}+J^{\mu} A_{\text {tot. } \mu}\right)
$$

where $g$ denotes the determinant of the matrix $\left(g_{\mu \nu}\right)$ of components of the metric, $J=J^{\mu} \partial_{\mu}$ is the 4 -current 'density' vector field, $A_{\text {tot. }}=A_{\text {tot. } \mu} \mathrm{d} x^{\mu}$ is the (tota $5^{5}$ electromagnetic 4-potential form and $\mathrm{d} A_{\text {tot. }}=F_{\text {tot. }}=F_{\text {tot. } \mu \nu} \mathrm{d} x^{\mu} \otimes \mathrm{d} x^{\nu}=\left(\partial_{\mu} A_{\text {tot. } \nu}-\right.$ $\left.\partial_{\nu} A_{\text {tot. } \mu}\right) \mathrm{d} x^{\mu} \otimes \mathrm{d} x^{\nu}$ is the electromagnetic field tensor. This is the standard action describing electromagnetism in a gravitational field, which is obtained by minimally coupling the special-relativistic action for electromagnetism [20] to a general spacetime metric [21, 22].

Note that $J^{\mu}$ are the components of a proper vector field and not of a density; their relation to the 4-current density with components $j^{\mu}$, in terms of which the interaction part of the action takes the form $\int \mathrm{d} t \mathrm{~d}^{3} \mathbf{x} j^{\mu} A_{\text {tot. } \mu}$, is given by

$$
J^{\mu}=\frac{1}{\sqrt{-g}} j^{\mu}
$$

\footnotetext{
${ }^{5}$ We will later decompose the field into internal and external contributions, hence the label 'total'.
} 
The current density of our system of two particles is given by ${ }^{6}$

$$
j^{\mu}(t, \mathbf{x})=\sum_{i=1}^{2} e_{i} \delta^{(3)}\left(\mathbf{x}-\mathbf{r}_{i}(t)\right) \dot{r}_{i}^{\mu}(t),
$$

where the dot denotes differentiation with respect to coordinate time $t$. The charge density is

$$
\rho:=\frac{1}{c} j^{0}
$$

The Maxwell equations obtained by varying the action with respect to $A_{\text {tot. } \mu}$ take the form

$$
\nabla_{\mu} F_{\text {tot. }}^{\mu \nu}=-\mu_{0} J^{\nu}
$$

in terms of the current vector field, or

$$
\nabla_{\mu} F_{\text {tot. }}^{\mu \nu}=-\mu_{0} \frac{1}{\sqrt{-g}} j^{\nu}
$$

in terms of the current density. It will be useful to consider the form

$$
\nabla^{\mu} F_{\text {tot. } \mu \nu}=-\mu_{0} \frac{1}{\sqrt{-g}} j_{\nu}
$$

instead.

From now on we employ the approximation that over the extension of the atom, the gravitational field $\phi$ is constant. This implies that all partial derivatives of the components of the metric vanish, such that the Christoffel symbols vanish and all covariant derivatives are given just by partial derivatives. Furthermore, we employ the Coulomb gauge $\nabla^{i} A_{\text {tot. } i}=0$. Then, the Maxwell equations 4.7 become

$$
-\mu_{0} \frac{1}{\sqrt{-g}} j_{\nu}=g^{\mu \rho} \partial_{\mu} \partial_{\rho} A_{\text {tot. } \nu}-g^{0 \rho} \partial_{\nu} \partial_{\rho} A_{\text {tot. } 0} \cdot
$$

We now split the electromagnetic potential into 'internal' and 'external' parts, as done in [1, i.e. the part generated by our system of moving particles and that corresponding to external electromagnetic fields: We have $A_{\text {tot. } \mu}=\mathcal{A}_{\mu}+A_{\mu}$, where we adopt the Coulomb gauge for both the external part $A_{\mu}$ and the internal part $\mathcal{A}_{\mu}$. The external potential $A_{\mu}$ is assumed as given and satisfying the vacuum Maxwell equations, and the internal potential satisfies the Maxwell equations for the internal current density (4.3), i.e.

$$
-\mu_{0} \frac{1}{\sqrt{-g}} j_{\nu}=g^{\mu \rho} \partial_{\mu} \partial_{\rho} \mathcal{A}_{\nu}-g^{0 \rho} \partial_{\nu} \partial_{\rho} \mathcal{A}_{0}
$$

\footnotetext{
${ }^{6}$ For a single particle of charge $q$ on an arbitrarily parametrised timelike worldline $r^{\mu}(\lambda)$, the current density is given by

$$
j^{\mu}(x)=q c \int \mathrm{d} \lambda \frac{\mathrm{d} r^{\mu}}{\mathrm{d} \lambda} \delta^{(4)}(x-r(\lambda)) .
$$

Parametrising by coordinate time and considering two particles, we arrive at the above expression.
} 
Similarly, we write $F_{\text {tot. } \mu \nu}=\mathcal{F}_{\mu \nu}+F_{\mu \nu}$, where $\mathcal{F}=\mathrm{d} \mathcal{A}$ is the internal and $F=\mathrm{d} A$ is the external field tensor.

Inserting the PPN metric (2.2), the 0-component of the Maxwell equations $(4.9)$ reads as follows:

$$
\begin{aligned}
-\mu_{0} \frac{1}{\sqrt{-g}} j_{0} & =g^{\mu \rho} \partial_{\mu} \partial_{\rho} \mathcal{A}_{0}-g^{0 \rho} \partial_{0} \partial_{\rho} \mathcal{A}_{0} \\
& =g^{a \rho} \partial_{a} \partial_{\rho} \mathcal{A}_{0} \\
& =c^{-1} g^{0 a} \partial_{t} \partial_{a} \mathcal{A}_{0}+g^{a b} \partial_{a} \partial_{b} \mathcal{A}_{0} \\
& =\left(1+2 \gamma \frac{\phi}{c^{2}}\right) \Delta \mathcal{A}_{0}+\mathrm{O}\left(c^{-4}\right)
\end{aligned}
$$

Here $\Delta=\delta^{a b} \partial_{a} \partial_{b}$ denotes the 'flat' spatial Laplace operator with respect to the spatial Euclidean metric defined by the background structures (flat Minkowski metric and preferred time-like vector field).

Now, using $71 / \sqrt{-g}=1+(3 \gamma-1) \frac{\phi}{c^{2}}+\mathrm{O}\left(c^{-4}\right)$, this equation is equivalent to

$$
\Delta \mathcal{A}_{0}=-\left(1+(\gamma-1) \frac{\phi}{c^{2}}\right) \mu_{0} j_{0}+\mathrm{O}\left(c^{-4}\right)
$$

Rewriting $j_{0}=-\left(1+2 \frac{\phi}{c^{2}}\right) j^{0}+\mathrm{O}\left(c^{-4}\right)$, recalling the charge density (4.4) and introducing the internal electric scalar potential

$$
\phi_{\text {el. }}:=-c \mathcal{A}_{0},
$$

we arrive at the Poisson equation for the electric potential:

$$
\Delta \phi_{\mathrm{el} .}=-\frac{1}{\varepsilon_{0}}\left(1+(\gamma+1) \frac{\phi}{c^{2}}\right) \rho+\mathrm{O}\left(c^{-3}\right) .
$$

The $a$ component of the Maxwell equations 4.9 is:

$$
\begin{aligned}
-\mu_{0} \frac{1}{\sqrt{-g}} j_{a} & =g^{\mu \rho} \partial_{\mu} \partial_{\rho} \mathcal{A}_{a}-g^{0 \rho} \partial_{a} \partial_{\rho} \mathcal{A}_{0} \\
& =g^{\mu \rho} \partial_{\mu} \partial_{\rho} \mathcal{A}_{a}-\left(1-2 \frac{\phi}{c^{2}}+\mathrm{O}\left(c^{-4}\right)\right) \varepsilon_{0} \mu_{0} \partial_{a} \partial_{t} \phi_{\mathrm{el}}
\end{aligned}
$$

With $g^{\mu \rho} \partial_{\mu} \partial_{\rho} \mathcal{A}_{a}=\left(1+2 \gamma \frac{\phi}{c^{2}}\right) \Delta \mathcal{A}_{a}-c^{-2} \partial_{t}^{2} \mathcal{A}_{a}+\mathrm{O}\left(c^{-4}\right)=\left(1+2 \gamma \frac{\phi}{c^{2}}\right)\left(\Delta-c^{-2} \partial_{t}^{2}\right) \mathcal{A}_{a}+$ $\mathrm{O}\left(c^{-4}\right)$, this is equivalent to

$$
\left(\Delta-c^{-2} \partial_{t}^{2}\right) \mathcal{A}_{a}=-\mu_{0}\left[\left(1+(\gamma-1) \frac{\phi}{c^{2}}\right) j_{a}-\left(1-2(\gamma+1) \frac{\phi}{c^{2}}\right) \varepsilon_{0} \partial_{a} \partial_{t} \phi_{\mathrm{el} .}\right]+\mathrm{O}\left(c^{-4}\right) .
$$

\footnotetext{
${ }^{7}$ The determinant of the spatial metric is $\operatorname{det}\left(g_{a b}\right)=:{ }^{(3)} g=1-6 \gamma \frac{\phi}{c^{2}}+\mathrm{O}\left(c^{-4}\right)$, and so minus the determinant of the metric is $-g={ }^{(3)} g /\left(-g^{00}\right)=\left(1-6 \gamma \frac{\phi}{c^{2}}+\mathrm{O}\left(c^{-4}\right)\right) \cdot\left(1+2 \frac{\phi}{c^{2}}+\mathrm{O}\left(c^{-4}\right)\right)=$ $1-2(3 \gamma-1) \frac{\phi}{c^{2}}+\mathrm{O}\left(c^{-4}\right)$, implying $\sqrt{-g}=1-(3 \gamma-1) \frac{\phi}{c^{2}}+\mathrm{O}\left(c^{-4}\right)$.
} 
Rewriting $j_{a}=\left(1-2 \gamma \frac{\phi}{c^{2}}\right) j^{a}+\mathrm{O}\left(c^{-4}\right)$ and introducing the internal vector potentia ${ }^{8}$ $\mathcal{A}=\mathcal{A}^{\perp}$ in $\mathcal{A}_{\mu}=\left(-\phi_{\text {el. }} / c, \mathcal{A}^{\perp}\right)$, the wave equation for this potential reads as follows:

$$
\left(\Delta-c^{-2} \partial_{t}^{2}\right) \mathcal{A}^{\perp}=-\mu_{0}\left[\left(1-(\gamma+1) \frac{\phi}{c^{2}}\right) \mathbf{j}-\left(1-2(\gamma+1) \frac{\phi}{c^{2}}\right) \varepsilon_{0} \nabla \partial_{t} \phi_{\text {el. }}\right]+\mathrm{O}\left(c^{-4}\right) .
$$

Now solving the equations 4.13) and 4.16) for the internal potentials as in appendix A of [1], we see that to leading post-Newtonian order we arrive at the same potentials as in the non-gravitational case, up to prefactors: We have

$$
\phi_{\mathrm{el} .}=\left(1+(\gamma+1) \frac{\phi}{c^{2}}\right) \phi_{\mathrm{el} ., \mathrm{ng}}
$$

and

$$
\mathcal{A}^{\perp}=\left(1-(\gamma+1) \frac{\phi}{c^{2}}\right) \mathcal{A}_{\mathrm{ng}}^{\perp}
$$

where the suffix 'ng' stands for 'non-gravitational', i.e. for the solutions from ([1].A1), ([1].A3). Since we are assuming the absence of external charges, the external electric scalar potential $A_{0}$ vanishes due to the 'external' equivalent of (4.10); and we introduce the external transverse vector potential $\mathbf{A}=\mathbf{A}^{\perp}$ as $A_{\mu}=\left(0, \mathbf{A}^{\perp}\right)$.

\subsection{Computation of the electromagnetic Lagrangian}

We will now use the obtained potentials to compute the electromagnetic Lagrangian

$$
L_{\mathrm{em}}=\int \mathrm{d}^{3} \mathbf{x}\left(-\frac{1}{4 \mu_{0}} \sqrt{-g} F_{\text {tot. } \mu \nu} F_{\text {tot. }}^{\mu \nu}+j^{\mu} A_{\text {tot. } \mu}\right)
$$

\footnotetext{
${ }^{8}$ It is a transverse vector field because of the Coulomb gauge condition.
} 
which follows from the action (4.1). For the internal kinetic Maxwell term, we obtain

$$
\begin{aligned}
-\frac{1}{4 \mu_{0}} \int \mathrm{d}^{3} \mathbf{x} \sqrt{-g} \mathcal{F}_{\mu \nu} \mathcal{F}^{\mu \nu}= & -\frac{1}{2 \mu_{0}} \int \mathrm{d}^{3} \mathbf{x} \sqrt{-g} \partial_{\mu} \mathcal{A}_{\nu} \mathcal{F}^{\mu \nu} \\
(\text { P.I. })= & \frac{1}{2 \mu_{0}} \int \mathrm{d}^{3} \mathbf{x} \sqrt{-g} \mathcal{A}_{\nu} \nabla_{\mu} \mathcal{F}^{\mu \nu} \\
& -\frac{1}{2 \mu_{0}} \int \mathrm{d}^{3} \mathbf{x}\left(\sqrt{-g} \partial_{0} \mathcal{A}_{\nu} \mathcal{F}^{0 \nu}+\mathcal{A}_{\nu} \partial_{0}\left(\sqrt{-g} \mathcal{F}^{0 \nu}\right)\right) \\
= & -\frac{1}{2} \int \mathrm{d}^{3} \mathbf{x} \mathcal{A}_{\nu} j^{\nu} \\
& -\frac{1}{2 \mu_{0}} \int \mathrm{d}^{3} \mathbf{x}\left(\sqrt{-g} \partial_{0} \mathcal{A}_{a}\left(\nabla^{0} \mathcal{A}^{a}-\nabla^{a} \mathcal{A}^{0}\right)\right. \\
& \left.+\mathcal{A}_{a} \partial_{0}\left(\sqrt{-g} \nabla^{0} \mathcal{A}^{a}-\sqrt{-g} \nabla^{a} \mathcal{A}^{0}\right)\right) \\
\left(\text { P.I., } \partial_{i} g_{\mu \nu}=0, \nabla^{i} \mathcal{A}_{i}=0\right)= & -\frac{1}{2} \int \mathrm{d}^{3} \mathbf{x} \mathcal{A}_{\nu} j^{\nu} \\
& -\frac{1}{2 \mu_{0}} \int \mathrm{d}^{3} \mathbf{x} \sqrt{-g}\left(\partial_{0} \mathcal{A}_{a} \partial^{0} \mathcal{A}^{a}+\mathcal{A}_{a} \partial_{0} \partial^{0} \mathcal{A}^{a}\right)+\mathrm{O}\left(c^{-4}\right)
\end{aligned}
$$

where in the last step, to perform the partial integration, we used that the metric is diagonal up to $\mathrm{O}\left(c^{-4}\right)$. Inserting the PPN metric, we obtain

$$
\begin{aligned}
& -\frac{1}{2 \mu_{0}} \int \mathrm{d}^{3} \mathbf{x} \sqrt{-g}\left(\partial_{0} \mathcal{A}_{a} \partial^{0} \mathcal{A}^{a}+\mathcal{A}_{a} \partial_{0} \partial^{0} \mathcal{A}^{a}\right) \\
& \quad=-\frac{\varepsilon_{0}}{2} \int \mathrm{d}^{3} \mathbf{x} \sqrt{-g} g^{00} g^{a b}\left(\partial_{t} \mathcal{A}_{a} \partial_{t} \mathcal{A}_{b}+\mathcal{A}_{a} \partial_{t}^{2} \mathcal{A}_{b}\right)+\mathrm{O}\left(c^{-4}\right) \\
& \quad=\left(1-(\gamma+1) \frac{\phi}{c^{2}}\right) \frac{\varepsilon_{0}}{4} \int \mathrm{d}^{3} \mathbf{x} \partial_{t}^{2} \mathcal{A}^{2}+\mathrm{O}\left(c^{-4}\right) .
\end{aligned}
$$

Thus, combining 4.20) and 4.21), the 'purely internal' contribution of electromagnetism to the Lagrangian, including the explicit coupling term of the internal potential to the current, is:

$$
\begin{aligned}
L_{\text {em }, \text { int. }} & =\int \mathrm{d}^{3} \mathbf{x}\left(-\frac{1}{4 \mu_{0}} \sqrt{-g} \mathcal{F}_{\mu \nu} \mathcal{F}^{\mu \nu}+j^{\mu} \mathcal{A}_{\mu}\right) \\
& =\frac{1}{2} \int \mathrm{d}^{3} \mathbf{x} j^{\mu} \mathcal{A}_{\mu}+\left(1-(\gamma+1) \frac{\phi}{c^{2}}\right) \frac{\varepsilon_{0}}{4} \int \mathrm{d}^{3} \mathbf{x} \partial_{t}^{2} \mathcal{A}^{2}+\mathrm{O}\left(c^{-4}\right)
\end{aligned}
$$

Following [1, appendix B], we will neglect the second integral in this expression ${ }^{9}$

For computing the other (purely external and mixed external-internal) contributions to the total electromagnetic Lagrangian (4.19), it is easiest to first rewrite the total

\footnotetext{
${ }^{9}$ Quoting [1, appendix B], it leads to terms 'proportional to the electrostatic energy of the atom divided by $m_{i} c^{2}$ times $\left|\mathbf{r}_{j}\right|^{2} / c^{2}$, which goes beyond our level of approximation'.
} 
kinetic Maxwell term. Using the PPN metric, but not the gauge condition, and writing $A_{\text {tot. } \mu}=\left(-\phi_{\text {el.,tot. }} / c, \mathbf{A}_{\text {tot. }}\right)$, we have:

$$
\begin{aligned}
&-\frac{1}{4 \mu_{0}} \sqrt{-g} F_{\text {tot. } \mu \nu} F_{\text {tot. }}^{\mu \nu} \\
&=\frac{\varepsilon_{0}}{2} \sqrt{-g}\left[-g^{00} g^{a b}\left(\partial_{t} A_{\text {tot. } a}+\partial_{a} \phi_{\text {el.,tot. }}\right)\left(\partial_{t} A_{\text {tot. } b}+\partial_{b} \phi_{\text {el.,tot. }}\right)\right. \\
&\left.\left.\quad-c^{2}\left(g^{a b} g^{c d}-g^{a d} g^{c b}\right) \partial_{a} A_{\text {tot. } .} \partial_{b} A_{\text {tot. } . d}\right)\right]+\mathrm{O}\left(c^{-4}\right) \\
&=\frac{\varepsilon_{0}}{2} {\left[\left(1-(\gamma+1) \frac{\phi}{c^{2}}\right)\left(\partial_{t} \mathbf{A}_{\text {tot. }}+\nabla \phi_{\text {el.,tot. }}\right)^{2}\right.} \\
&\left.\quad-c^{2}\left(1+(\gamma+1) \frac{\phi}{c^{2}}\right)\left(\nabla \times \mathbf{A}_{\text {tot. }}\right)^{2}\right]+\mathrm{O}\left(c^{-4}\right)
\end{aligned}
$$

We recall that, as explained in section 2.3 , the boldface nabla symbol $\boldsymbol{\nabla}$ denotes the three-tuple of partial derivatives, and $\boldsymbol{\nabla} \times \mathbf{A}_{\text {tot. }}$ denotes the 'component-wise curl' of $\mathbf{A}_{\text {tot. }}$. Both of these operations are well-defined (independent of coordinates) once we have introduced the background structures (flat Minkowski metric and preferred time-like vector field).

The internal-internal term of 4.23 was considered above in 4.22). The externalexternal term gives ( $\phi_{\text {el.,tot. }}$ is internal, and $\mathbf{A}=\mathbf{A}^{\perp}$ is transverse because of the gauge condition)

$$
\begin{aligned}
L_{\text {em }, \text { ext. }}= & -\frac{1}{4 \mu_{0}} \int \mathrm{d}^{3} \mathbf{x} \sqrt{-g} F_{\mu \nu} F^{\mu \nu} \\
= & \frac{\varepsilon_{0}}{2} \int \mathrm{d}^{3} \mathbf{x}\left[\left(1-(\gamma+1) \frac{\phi}{c^{2}}\right)\left(\partial_{t} \mathbf{A}^{\perp}\right)^{2}\right. \\
& \left.-c^{2}\left(1+(\gamma+1) \frac{\phi}{c^{2}}\right)\left(\boldsymbol{\nabla} \times \mathbf{A}^{\perp}\right)^{2}\right]+\mathrm{O}\left(c^{-4}\right),
\end{aligned}
$$

and the external-internal mixed term plus the interaction of the external potential with the current is (using partial integration and the gauge condition to get rid of the $\partial_{t} \mathbf{A}^{\perp} \cdot \nabla \phi_{\text {el. }}$ term)

$$
\begin{aligned}
L_{\text {em,ext.-int. }}= & \int \mathrm{d}^{3} \mathbf{x} j^{\mu} A_{\mu}-\frac{1}{2 \mu_{0}} \int \mathrm{d}^{3} \mathbf{x} \sqrt{-g} \mathcal{F}_{\mu \nu} F^{\mu \nu} \\
= & \int \mathrm{d}^{3} \mathbf{x} j^{\mu} A_{\mu}+\varepsilon_{0} \int \mathrm{d}^{3} \mathbf{x}\left[\left(1-(\gamma+1) \frac{\phi}{c^{2}}\right)\left(\partial_{t} \mathcal{A}^{\perp}\right) \cdot\left(\partial_{t} \mathbf{A}^{\perp}\right)\right. \\
& \left.-c^{2}\left(1+(\gamma+1) \frac{\phi}{c^{2}}\right)\left(\boldsymbol{\nabla} \times \mathcal{A}^{\perp}\right) \cdot\left(\boldsymbol{\nabla} \times \mathbf{A}^{\perp}\right)\right]+\mathrm{O}\left(c^{-4}\right) .
\end{aligned}
$$

Following [1, appendix B], we will neglect the second integral also in this expression, since it is related to formally diverging backreaction terms. 
Adding the Lagrangians 4.22, 4.24) and 4.25), the total post-Newtonian electromagnetic Lagrangian (with the above-mentioned neglections following [1]) reads:

$$
\begin{aligned}
L_{\mathrm{em}}= & \frac{1}{2} \int \mathrm{d}^{3} \mathbf{x}\left(\mathbf{j} \cdot \mathcal{A}^{\perp}-\rho \phi_{\mathrm{el} .}\right)+\int \mathrm{d}^{3} \mathbf{x} \mathbf{j} \cdot \mathbf{A}^{\perp} \\
& +\frac{\varepsilon_{0}}{2} \int \mathrm{d}^{3} \mathbf{x}\left[\left(1-(\gamma+1) \frac{\phi}{c^{2}}\right)\left(\partial_{t} \mathbf{A}^{\perp}\right)^{2}-c^{2}\left(1+(\gamma+1) \frac{\phi}{c^{2}}\right)\left(\boldsymbol{\nabla} \times \mathbf{A}^{\perp}\right)^{2}\right]+\mathrm{O}\left(c^{-4}\right)
\end{aligned}
$$

Inserting the internal potentials (4.17), (4.18) as well as the current and charge densities (4.3), (4.4) and dropping infinite self-interaction terms for the internal part of the electromagnetic Lagrangian we obtain

$$
\begin{aligned}
& \frac{1}{2} \int \mathrm{d}^{3} \mathbf{x}\left(\mathbf{j} \cdot \mathcal{A}^{\perp}-\rho \phi_{\mathrm{el} .}\right) \\
&=-\left(1+(\gamma+1) \frac{\phi}{c^{2}}\right) \frac{e_{1} e_{2}}{4 \pi \varepsilon_{0} r} \\
&+\left(1-(\gamma+1) \frac{\phi}{c^{2}}\right) \frac{e_{1} e_{2}}{8 \pi \varepsilon_{0} c^{2}}\left[\frac{\dot{\mathbf{r}}_{1} \cdot \dot{\mathbf{r}}_{2}}{r}+\frac{\left(\dot{\mathbf{r}}_{1} \cdot \mathbf{r}\right)\left(\dot{\mathbf{r}}_{2} \cdot \mathbf{r}\right)}{r^{3}}\right]+\mathrm{O}\left(c^{-4}\right) \\
&=-\left(1+(\gamma+1) \frac{\phi}{c^{2}}\right) \frac{e_{1} e_{2}}{4 \pi \varepsilon_{0} r}+\frac{e_{1} e_{2}}{8 \pi \varepsilon_{0} c^{2}}\left[\frac{\dot{\mathbf{r}}_{1} \cdot \dot{\mathbf{r}}_{2}}{r}+\frac{\left(\dot{\mathbf{r}}_{1} \cdot \mathbf{r}\right)\left(\dot{\mathbf{r}}_{2} \cdot \mathbf{r}\right)}{r^{3}}\right]+\mathrm{O}\left(c^{-4}\right) .
\end{aligned}
$$

\section{The total Hamiltonian including all interactions}

In this section we collect all previous findings and combine them into the total Hamiltonian that characterises the dynamics of our two-particle system that is now also exposed to a non-trivial gravitational field. We will see that the Hamiltonian suffers various 'corrections' as compared to the gravity-free case, and that these terms acquire an intuitive interpretation if re-expressed in terms of the physical spacetime metric $g$.

\subsection{Computation of the Hamiltonian}

We will now compute the total Hamiltonian describing the atom in external electromagnetic and gravitational fields by repeating the calculation from section 3 while including the 'gravitational corrections' to electromagnetism obtained in section 4 .

Comparing the gravitationally corrected electromagnetic Lagrangian (4.26), (4.27) to the one without gravitational field $(\phi=0)$, we see that at our order of approximation the only differences occur in the external electromagnetic term and the internal Coulomb interaction term. Thus, when calculating the Hamiltonian, we have to only take care of these changes compared to the discussion of section 3 .

The canonical momentum conjugate to $\mathbf{A}^{\perp}$ is

$$
\boldsymbol{\Pi}^{\perp}=\frac{\delta L_{\mathrm{em}}}{\delta\left(\partial_{t} \mathbf{A}^{\perp}\right)}=\varepsilon_{0}\left(1-(\gamma+1) \frac{\phi}{c^{2}}\right) \partial_{t} \mathbf{A}^{\perp}+\mathrm{O}\left(c^{-4}\right),
$$


and the Hamiltonian for the external electromagnetic field thus is

$$
\begin{aligned}
H_{\text {em }, \text { ext. }} & =\int \mathrm{d}^{3} \mathbf{x} \boldsymbol{\Pi}^{\perp} \cdot \partial_{t} \mathbf{A}^{\perp}-L_{\text {em,ext }} \\
& =\frac{\varepsilon_{0}}{2} \int \mathrm{d}^{3} \mathbf{x}\left(1+(\gamma+1) \frac{\phi}{c^{2}}\right)\left[\left(\boldsymbol{\Pi}^{\perp} / \varepsilon_{0}\right)^{2}+c^{2}\left(\boldsymbol{\nabla} \times \mathbf{A}^{\perp}\right)^{2}\right]+\mathrm{O}\left(c^{-4}\right) .
\end{aligned}
$$

Thus, when including the new corrections, the classical Hamiltonian will be the same as

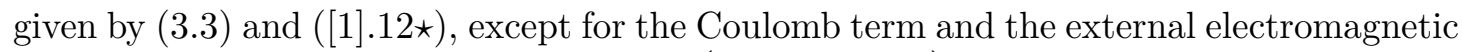
field energy both gaining the prefactor $\left(1+(\gamma+1) \frac{\phi}{c^{2}}\right)$. The same will be true after quantising and performing the PZW transformation and electric dipole approximation: Using the same expression $([1] .14 \star)$ for the PZW operator, we arrive at the multipolar

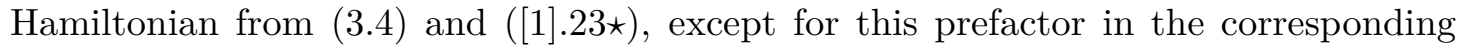
terms.

Now transforming to central and relative coordinates, as in going from (3.4) and $([1] .23 \star)$ to $(3.9)$ and $([1] .25 \star)$, the same stays true; we just have to keep in mind that the relation between the electromagnetic canonical momentum $\Pi^{\perp}$ and the transverse external 'coordinate electric field' $\mathbf{E}_{\text {coord. }}^{\perp}=-\partial_{t} \mathbf{A}^{\perp}$ now also gains an additional factor. Including this, the electric field-dipole interaction term again has the form $-\mathbf{d} \cdot \mathbf{E}_{\text {coord. }}^{\perp}(\mathbf{R})$ as in the non-gravitational case, but the dipole polarisation self-interaction term $\frac{1}{2 \varepsilon_{0}} \int \mathrm{d}^{3} \mathbf{x} \mathcal{P}_{d}^{\perp}{ }^{2}$ in $H_{\mathrm{AL}}$ gains a prefactor (since it arises from the PZW-transformed external field energy).

Thus, comparing to the discussion of section 3 , the external electromagnetic field energy, the $\mathcal{P}_{d}^{\perp^{2}}$ term in $H_{\mathrm{AL}}$ and the Coulomb term are multiplied by the factor $\left(1+(\gamma+1) \frac{\phi}{c^{2}}\right)$, and we arrive at the following total Hamiltonian:

$$
\begin{aligned}
H_{\text {[com],final }} & =H_{\mathrm{C}, \text { final }}+H_{\mathrm{A}, \text { final }}+H_{\mathrm{AL}, \text { final }}+H_{\mathrm{L}, \text { final }}+H_{\mathrm{X}} \\
H_{\mathrm{C}, \text { final }} & =H_{\mathrm{C}, \text { new }}-\frac{1}{c^{2}} \frac{e^{2}}{4 \pi \varepsilon_{0} r} \phi(\mathbf{R}) \\
H_{\mathrm{A}, \text { final }} & =H_{\mathrm{A}, \text { new }}-\gamma \frac{1}{c^{2}} \frac{e^{2}}{4 \pi \varepsilon_{0} r} \phi(\mathbf{R}) \\
H_{\mathrm{AL}, \text { final }} & =H_{\mathrm{AL}}+\frac{1}{2 \varepsilon_{0}} \int \mathrm{d}^{3} \mathbf{x}(\gamma+1) \frac{\phi}{c^{2}} \boldsymbol{\mathcal { P }}_{d}^{\perp^{2}} \\
H_{\mathrm{L}, \text { final }} & =\frac{\varepsilon_{0}}{2} \int \mathrm{d}^{3} \mathbf{x}\left(1+(\gamma+1) \frac{\phi}{c^{2}}\right)\left[\left(\mathbf{\Pi}^{\perp} / \varepsilon_{0}\right)^{2}+c^{2}\left(\boldsymbol{\nabla} \times \mathbf{A}^{\perp}\right)^{2}\right]
\end{aligned}
$$

Here, we have included the term $-\gamma \frac{1}{c^{2}} \frac{e^{2}}{4 \pi \varepsilon_{0} r} \phi(\mathbf{R})$ into $H_{\mathrm{A}, \text { final }}$ since it can be combined with the original Coulomb term from $H_{\mathrm{A}}$ into

$$
-\frac{e^{2}}{4 \pi \varepsilon_{0} r}\left(1+\gamma \frac{\phi(\mathbf{R})}{c^{2}}\right)=-\frac{e^{2}}{4 \pi \varepsilon_{0} \sqrt{{ }^{(3)} g_{\mathbf{R}}(\mathbf{r}, \mathbf{r})}},
$$

i.e. a Coulomb term expressed with the correct, metric relative distance.

Note that we neglected the terms $\mathbf{r} \cdot \boldsymbol{\nabla} \phi(\mathbf{R})$ (since we assumed $\phi$ to be constant over the extension of the atom), thus arriving at the same cross terms $H_{\mathrm{X}}$ as in [1].25f; similarly, the corresponding term from $H_{\mathrm{A} \text {,new }}(3.12)$ can be neglected. 
To correctly interpret the atom-light interaction Hamiltonian (5.3d), one has to keep in mind that the field variables $\mathbf{E}^{\perp}$ and $\mathbf{B}$ in $H_{\mathrm{AL}}([1] .25 \mathrm{~d} \star)$ are the coordinate components $-\partial_{t} \mathbf{A}^{\perp}$ and $\boldsymbol{\nabla} \times \mathbf{A}^{\perp}$ respectively, which do not refer to an orthonormal frame in the physical spacetime metric $g$ in the presence of gravitational fields. This issue will be discussed in more detail in section 5.3 .

Since the cross terms $H_{\mathrm{X}}$ are the same as in [1], we could now introduce new coordinates $\mathbf{Q}, \mathbf{q}, \mathbf{p}$ literally as in $(1,26$ to eliminate these cross terms. Since the gravitational correction terms are of order $\mathrm{O}\left(c^{-2}\right)$, for them this change into the new coordinates would just amount to the replacements $\mathbf{R} \rightarrow \mathbf{Q}, \mathbf{r} \rightarrow \mathbf{q}, \mathbf{p}_{\mathbf{r}} \rightarrow \mathbf{p}$ at our order of approximation. Since it will not alter the following discussion, we will not perform this coordinate change in order to avoid adding an extra layer of potentially confusing notation.

\subsection{The system as a composite point particle}

Fully writing out the central and internal Hamiltonian $(5.3 \mathrm{~b}),(5.3 \mathrm{c})$, we obtain

$$
\begin{aligned}
H_{\mathrm{C}, \text { final }}= & \frac{\mathbf{P}^{2}}{2 M}\left[1-\frac{1}{M c^{2}}\left(\frac{\mathbf{p}_{\mathbf{r}}^{2}}{2 \mu}+\frac{e_{1} e_{2}}{4 \pi \varepsilon_{0} r}\right)\right]+\left[M+\frac{1}{c^{2}}\left(\frac{\mathbf{p}_{\mathbf{r}}^{2}}{2 \mu}+\frac{e_{1} e_{2}}{4 \pi \varepsilon_{0} r}\right)\right] \phi(\mathbf{R}) \\
& -\frac{\mathbf{P}^{4}}{8 M^{3} c^{2}}+\frac{2 \gamma+1}{2 M c^{2}} \mathbf{P} \cdot \phi(\mathbf{R}) \mathbf{P}+(2 \beta-1) \frac{M \phi(\mathbf{R})^{2}}{2 c^{2}}, \\
H_{\mathrm{A}, \text { final }}= & \frac{(3) g_{\mathbf{R}}^{-1}\left(\mathbf{p}_{\mathbf{r}}, \mathbf{p}_{\mathbf{r}}\right)}{2 \mu}+\frac{e_{1} e_{2}}{4 \pi \varepsilon_{0} \sqrt{(3)} g_{\mathbf{R}(\mathbf{r}, \mathbf{r})}} \\
& -\frac{m_{1}^{3}+m_{2}^{3}}{M^{3}} \frac{\mathbf{p}_{\mathbf{r}}^{4}}{8 \mu^{3} c^{2}}+\frac{e_{1} e_{2}}{4 \pi \varepsilon_{0}} \frac{1}{2 \mu M c^{2}}\left(\mathbf{p}_{\mathbf{r}} \cdot \frac{1}{r} \mathbf{p}_{\mathbf{r}}+\mathbf{p}_{\mathbf{r}} \cdot \mathbf{r} \frac{1}{r^{3}} \mathbf{r} \cdot \mathbf{p}_{\mathbf{r}}\right),
\end{aligned}
$$

where we combined the gravitational correction terms in $H_{\mathrm{A} \text {,final }}$ into metrically defined kinetic energy and Coulomb terms as in (3.13), (5.4).

Comparing to the Hamiltonian of a single point particle of mass $m$ in the PPN metric,

$$
H_{\text {point }}(\mathbf{P}, \mathbf{R} ; m)=\frac{\mathbf{P}^{2}}{2 m}+m \phi(\mathbf{R})-\frac{\mathbf{P}^{4}}{8 m^{3} c^{2}}+\frac{2 \gamma+1}{2 m c^{2}} \mathbf{P} \cdot \phi(\mathbf{R}) \mathbf{P}+(2 \beta-1) \frac{m \phi(\mathbf{R})^{2}}{2 c^{2}},
$$

we thus see that the central Hamiltonian has, up to (and including) $\mathrm{O}\left(c^{-2}\right)$, exactly this form, with the mass $m$ replaced by $M+\frac{H_{\mathrm{A}, \text { final }}}{c^{2}}$,

$$
H_{\mathrm{C}, \text { final }}=H_{\text {point }}\left(\mathbf{P}, \mathbf{R} ; M+\frac{H_{\mathrm{A}, \text { final }}}{c^{2}}\right)
$$

as could be naively expected from mass-energy equivalence. Thus, starting from first principles, we have shown that the system behaves as a 'composite point particle' whose (inertial as well as gravitational) mass is comprised of the rest masses of the constituent particles as well as the internal energy.

Note that this conclusion depends on the identification of terms as being 'kinetic' and 'interaction' energies, which in turn depends on the metric structure in their expressions. Had we not rewritten the internal kinetic energy 3.13 and the Coulomb interaction 5.4 
in terms of the physical metric $g$, the above conclusion could not have resulted. Rather, we would have had to replace the inertial mass of the 'composite particle' by $M+\frac{H_{\mathrm{A}}}{c^{2}}$ and the gravitational mass by $M+(2 \gamma+1) \frac{\mathbf{p}^{2}}{2 \mu c^{2}}+(\gamma+1) \frac{e_{1} e_{2}}{4 \pi \varepsilon_{0} q c^{2}}=M+\frac{H_{\mathrm{A}}}{c^{2}}+\gamma\left(2 \frac{\mathbf{p}^{2}}{2 \mu c^{2}}+\frac{e_{1} e_{2}}{4 \pi \varepsilon_{0} q c^{2}}\right)$, which one could have erroneously interpreted as a violation of some naive form of the weak Equivalence Principle. But, clearly, such a conclusion would be premature, for it is based on the identification of terms - like inertial and gravitational mass - that is itself ambiguous. That ambiguity is here seen as a dependence on the background structure, which is used to define distances of positions and squares of momenta. Once these quantities are measured with the physical metric $g$, ambiguities and apparent conflicts with naive expectations disappear. That point has also been made in [8].

The quantities $\vec{p}^{2}$ and $r^{\prime}$ entering the Hamiltonian in [8, eq. (18)], which are, in the language of [8], the square of the internal momentum and the distance 'in the CM rest frame', are nothing but the geometric expressions ${ }^{(3)} g_{\mathbf{R}}^{-1}\left(\mathbf{p}_{\mathbf{r}}, \mathbf{p}_{\mathbf{r}}\right)$ and $\sqrt{{ }^{(3)} g_{\mathbf{R}}(\mathbf{r}, \mathbf{r})}$ from above, measured using the physical metric of space. The internal Hamiltonian (5.6) thus consists of kinetic and Coulomb interaction energies in terms of the physical geometry, in agreement with the expressions from [8], as well as the expected special-relativistic and 'Darwin' correction:10

\subsection{The electromagnetic expressions in terms of components with respect to orthonormal frames}

The expressions derived above in (5.3) include components of the electromagnetic field with respect to coordinates which, albeit not chosen arbitrarily, have no direct metric significance. We recall that we used coordinates that are adapted to the chosen background structure $(\eta, u)$. This means that $u=\partial / \partial t$ and $\eta=\eta_{\mu \nu} \mathrm{d} x^{\mu} \otimes \mathrm{d} x^{\nu}$ with $\left(\eta_{\mu \nu}\right)=$ $\operatorname{diag}(-1,1,1,1)$. The corresponding local reference frames $\left\{\partial_{\mu}:=\partial / \partial x^{\mu} \mid \mu=0,1,2,3\right\}$ are orthonormal with respect to $\eta$, but not with respect to the physical metric $g$.

In this subsection we will re-express our findings in terms of components with respect to orthonormal frames with respect to $g$, which we will call the 'physical components', as opposed to the 'coordinate components' used so far. We stress that, despite this terminology, there is nothing wrong or 'unphysical' with representing fields in terms of components of non orthonormal bases, as long as the metric properties are spelled out at the same time. Yet it is clearly convenient to be able to read off metric properties, which bear direct physical significance, from the expressions involving the components alone, without at the same time having to recall the values of the metric components as well.

\footnotetext{
${ }^{10}$ Since these corrections are themselves of order $1 / c^{2}$, the deviations of the physical from the flat metric do not enter here.
} 
The full atom-light interaction Hamiltonian $H_{\mathrm{AL}, \text { final }}$ (5.3d) reads

$$
\begin{aligned}
H_{\mathrm{AL}, \text { final }}= & -\mathbf{d} \cdot \mathbf{E}_{\text {coord. }}^{\perp}(\mathbf{R})+\frac{1}{2 M}\left\{\mathbf{P} \cdot\left[\mathbf{d} \times \mathbf{B}_{\text {coord. }}(\mathbf{R})\right]+\text { H.c. }\right\} \\
& -\frac{m_{1}-m_{2}}{4 m_{1} m_{2}}\left\{\mathbf{p}_{\mathbf{r}} \cdot\left[\mathbf{d} \times \mathbf{B}_{\text {coord. }}(\mathbf{R})\right]+\text { H.c. }\right\} \\
& +\frac{1}{8 \mu}\left(\mathbf{d} \times \mathbf{B}_{\text {coord. }}(\mathbf{R})\right)^{2}+\frac{1}{2 \varepsilon_{0}} \int \mathrm{d}^{3} \mathbf{x}\left(1+(\gamma+1) \frac{\phi}{c^{2}}\right) \mathcal{P}_{d}^{\perp}(\mathbf{x}, t),
\end{aligned}
$$

where $\mathbf{E}_{\text {coord. }}^{\perp}=-\partial_{t} \mathbf{A}^{\perp}$ and $\mathbf{B}_{\text {coord. }}^{\perp}=\nabla \times \mathbf{A}^{\perp}$, i.e. $E_{\text {coord. } a}=c F_{a 0}$ and $B_{\text {coord. }}^{a}=\varepsilon^{a b c} F_{b c}$, are given by the coordinate components of the electromagnetic field tensor that refer to non orthonormal frames $\partial_{\mu}$. Since our metrics $\eta$ and $g$ are both diagonal in the coordinates used and to the order of approximation employed here, we simply need to divide $\partial_{\mu}$ by the square-root of the modulus of $g_{\mu \mu}$ (no summation) in order to get an orthonormal frame $\mathrm{e}_{\underline{\mu}}$ for $g$, called a 'tetrad':

$$
\begin{gathered}
\mathrm{e}_{\underline{0}}=\frac{1}{\sqrt{-g_{00}}} \partial_{0}=\left(1-\frac{\phi}{c^{2}}\right) \partial_{0}, \\
\mathrm{e}_{\underline{a}}=\frac{1}{\sqrt{g_{a a}}} \partial_{a}=\left(1+\gamma \frac{\phi}{c^{2}}\right) \partial_{a}
\end{gathered}
$$

Note that from now on underlined indices refer to the tetrad ('physical components'), non underlined ones to the coordinate basis ('coordinate components').

The 'physical components' of the electric and magnetic fields with respect to the tetrad are given in terms of the tetrad components of the field tensor through

$$
E_{\text {phys } . \underline{a}}=c F_{\underline{a} \underline{0}}, \quad B_{\text {phys. }}^{\underline{a}}=\sum_{b, c=1}^{3} \varepsilon^{a b c} F_{\underline{b c}},
$$

where $\varepsilon^{a b c}$ is the totally antisymmetric symbol. This can be understood in a more geometric way: Denoting by $\tilde{\varepsilon}^{a b c}$ the tetrad components of the spatial volume form $\tilde{\varepsilon}$ that is induced by the physical metric $g$ (instead of the spatial volume form $\varepsilon$ induced by the background metric $\eta$ ), we have the numerical identity

$$
\tilde{\varepsilon}^{a b c}=\varepsilon^{a b c}
$$

since the tetrad basis vectors $\left\{\mathrm{e}_{\underline{a}}\right\}$ are orthonormal with respect to $g$. Thus, the magnetic field components can be written as

$$
B_{\text {phys. }}^{\underline{a}}=\tilde{\varepsilon}^{a b c} F_{\underline{b c}},
$$

with clear geometric meaning. Inserting the tetrad (5.10), the components (5.11) are related to the coordinate expressions $E_{\text {coord. } a}=c F_{a 0}$ and $B_{\text {coord. }}^{a}=\varepsilon^{a b c} F_{b c}$ by

$$
\mathbf{E}_{\text {phys. }}=\frac{1}{\sqrt{-g_{00}}}\left(1+\gamma \frac{\phi}{c^{2}}\right) \mathbf{E}_{\text {coord. }}, \mathbf{B}_{\text {phys. }}=\left(1+2 \gamma \frac{\phi}{c^{2}}\right) \mathbf{B}_{\text {coord. . }}
$$


Similarly, the dipole moment $d^{a}=\sum_{k=1,2} e_{k}\left(r_{k}^{a}-R^{a}\right)$ is defined via coordinate distances, and thus the physically significant, metric dipole moment is given by

$$
d_{\text {phys. }}^{\underline{a}}=\mathrm{e}_{b}^{a} d^{b}=\left(1-\gamma \frac{\phi}{c^{2}}\right) d^{a} .
$$

Thus the electric dipole interaction term in the Hamiltonian takes the form

$$
-\mathbf{d} \cdot \mathbf{E}_{\text {coord. }}^{\perp}(\mathbf{R})=-\sqrt{-g_{00}(\mathbf{R})} \mathbf{d}_{\text {phys. }} \cdot \mathbf{E}_{\text {phys. }}^{\perp}(\mathbf{R})
$$

when expressed in terms of physical components. The 'gravitational time dilation' factor $\sqrt{-g_{00}}$ in this expression could now also be absorbed by referring the time evolution to the proper time of the observer situated at $\mathbf{R}$ instead of coordinate time [9, 18].

Similarly, all the other interaction terms from $(5.9)$ can be rewritten in terms of tetrad components. The only difficulty arises when considering the Röntgen term, i.e. the second term in the interaction Hamiltonian, since it involves the momentum $\mathbf{P}$ : If the components $P_{a}$ were just the components of a classical covector field (i.e. a one-form), there would be no problem in computing its tetrad components as

$$
P_{\text {phys. } \underline{a}}=\mathrm{e}_{\underline{a}}^{b} P_{b}=\left(1-\gamma \frac{\phi}{c^{2}}\right) P_{a} .
$$

However, the $P_{a}$ are operators that don't commute with the centre of mass position $\mathbf{R}$, such that in the application of 5.17) one has to deal with with operator ordering ambiguities (which is, of course, a well-known issue regarding curvilinear coordinate transformations in Quantum Mechanics). Of course, to avoid dealing with these ambiguities, one can stay with the coordinate components of the momentum and rewrite only the other quantities in terms of tetrad components, arriving at

$$
\frac{1}{2 M}\left\{\mathbf{P} \cdot\left[\mathbf{d} \times \mathbf{B}_{\text {coord. }}(\mathbf{R})\right]+\text { H.c. }\right\}=\frac{1}{2 M}\left\{\mathbf{P} \cdot\left(1-\gamma \frac{\phi(\mathbf{R})}{c^{2}}\right)\left[\mathbf{d}_{\text {phys. }} \times \mathbf{B}_{\text {phys. }}(\mathbf{R})\right]+\text { H.c. }\right\}
$$

for the Röntgen term. When doing so, to give a well-defined geometric meaning to the resulting expression on the right-hand side, one has to keep in mind that the components $\mathbf{P}$ of the momentum refer to the coordinate basis and the components $\mathbf{d}_{\text {phys. }}, \mathbf{B}_{\text {phys. }}$ of the dipole moment and the magnetic field refer to the tetrad.

Note that for the internal momentum $\mathbf{p}_{\mathbf{r}}$, occurring in the third interaction term in (5.9), such operator ordering ambiguities do not arise, since we assumed the gravitational potential $\phi$ to be constant over the extension of the atom.

To the best of our knowledge, the atom-light interaction terms in the presence of gravity obtained in (5.9) and discussed above are new, save for the electric dipole coupling which was already discussed in [9, 18].

Finally, expressing the external field energy $(5.3 \mathrm{e})$ in terms of tetrad components, we obtain

$$
\begin{aligned}
H_{\mathrm{L}, \text { final }} & =\frac{\varepsilon_{0}}{2} \int \mathrm{d}^{3} \mathbf{x}\left(1+(1-3 \gamma) \frac{\phi}{c^{2}}\right)\left[\mathbf{E}_{\text {phys. }}^{\perp}+c^{2} \mathbf{B}_{\text {phys. }}^{2}\right] \\
& =\frac{\varepsilon_{0}}{2} \int \mathrm{d}^{3} \mathbf{x} \sqrt{-g}\left[\mathbf{E}_{\text {phys. }}^{\perp}{ }^{2}+c^{2} \mathbf{B}_{\text {phys. }}^{2}\right]
\end{aligned}
$$


which is the standard result of the flat-spacetime electromagnetic field energy [20] minimally coupled to gravity [21, as was to be expected.11]

\section{A comment on the Equivalence Principle in Quantum Mechanics}

As advertised at the end of the introduction, we now wish to come back to the less technical but conceptually all-important issue surrounding the meaning of 'Equivalence Principle', in particular concerning its susceptibility to be more accurately tested by genuine quantum matter. Clearly, that possibility presupposes the formulation of the Equivalence Principle in a way that is fully compatible with Quantum Mechanics. Our discussion here is motivated by our impression that this need, simple and obvious as it might seem to be, is often unduly neglected. As emphasised before, our central point of concern results from our conviction that any generally valid implementation of the Equivalence Principle into Quantum Mechanics should not be based on a priori assumptions concerning the state of the matter. In particular, it should not rely on semi-classical notions like 'wordline', that often enter discussions in a fundamental way ${ }^{12}$, see, e.g., [3-8]. It should also not rely on other classical formulations, like 'equality of inertial and gravitational mass', for reasons discussed below.

Rather, the meaning of the Equivalence Principle is to ensure that a single spacetime geometry accounts for all matter couplings, thereby comprising all gravity phenomena on all kinds of matter; compare [10. This does clearly not imply that all 'things' (bodies, wave packets, ... ) 'fall' with the same acceleration. First, in order to give a measure for the acceleration of 'fall', we need to kinematically define that term; that is, we need to specify a structure on which we can read-off such quantities as acceleration. In other words: we need to define a worldline. As discussed before, quantum-mechanical systems only do that in very special states. Second, even in case a worldline is approximately definable, there is no reason why it must coincide with that of another system if both start out in the same centre of mass initial state. This is not even true in classical physics. The universality of free fall reduces to the equality of worldlines only in case of 'test particles', idealised objects of partially opposing properties 13 For realistic bodies, which are spinning and/or possess higher mass multipoles, the centres of mass will not define universal point-particle trajectories (i.e. geodesics). To find the equations of motion for

\footnotetext{
${ }^{11}$ This result would have been immediate if we did the whole calculation in terms of tetrad components instead of coordinate components, as would have some steps in the calculation of the electromagnetic Lagrangian. However, as stressed in section 2.3 the approach based on the background structures enabled us to provide a direct comparison with the original calculation of 1 .

${ }^{12}$ In that respect we fully agree with $[23]$.

${ }^{13} \mathrm{~A}$ 'test particle' must have negligible size as compared to the curvature radius of the background geometry, so as to not couple to second and higher derivatives of the metric (i.e. no curvature couplings). At the same time, it cannot be too small, so as to not create a significant gravitational selfenergy and an appreciable self-gravitational field on top of that of background spacetime. Moreover, it may have no non-vanishing charges, spin, or higher moments amongst its mass multipoles. This should make it clear that the notion of 'test particle' is contextual and approximative. One and the same object may be a good test particle in one situation and badly fail to be such in another.
} 
structured bodies in arbitrary gravitational fields is, in fact, one of the most difficult challenges in General Relativity research [24. However, as long as all deviations from the geodesic motion find their explanations in couplings to the spacetime geometry, no violation of the Equivalence Principle should be concluded. Likewise, extended wave packets in Quantum Mechanics will move in a way that depends on the higher moments of their probability distribution [25], but this does not contradict any Equivalence Principle requirement, contrary to the impression given in [25]. In that context we wish to remind the reader of our discussion in the introduction.

Finally, we also wish to comment on attempts to translate the equality of inertial and gravitational (more precisely: passive gravitational) mass into Quantum Mechanics; see, e.g., [26]. These attempts also bear the danger to prematurely conclude apparent violations, even though all the couplings are through the geometry only. In fact, it seems to us that those attempts suffer from a logical flaw that we now wish to briefly characterise.

First the positive results: It is an elementary theorem in ordinary Quantum Mechanics that, given a spatially homogeneous but arbitrarily time dependent force field, any solution to the Schrödinger equation in that field corresponds to a solution of the forcefree Schrödinger equation written in the coordinates of a frame that freely falls according to the classical equations of motion in the given force field. This is, e.g., proven in [27]. If the force field is that of Newtonian gravity, such that the classical trajectory only depends on the ratio of the inertial to the gravitational mass, then the motion of any wave packet is that of a free Schrödinger wave in a universally (i.e. the same for all wave functions and all particles with the same ratio of inertial and gravitational mass) falling frame. In that sense there is no observational difference between a falling wave packet in a homogeneous gravitational field and a free wave packet without gravity viewed from a frame that rigidly moves with equal but opposite acceleration to that determined by the gravity field. This precisely corresponds to the Equivalence Principle for homogeneous fields in Newtonian gravity which we therefore see to extend to Quantum Mechanics.

In Classical Mechanics, we can extend the universality of free fall to non-homogeneous fields if we restrict attention to pointlike test particles. Their equation of motion then guarantees the universality of free fall, given that their inertial equals their gravitational mass. This shows that we need the particular equations of motion to provide the logical link between the universality of free fall on one side and the equality of the inertial and gravitational mass on the other. As such the latter equality does not tell us anything if this link is not provided. That, in our opinion, is the flaw in any premature identification of the weak Equivalence Principle with the equality of inertial and gravitational mass (leaving alone the ambiguity to define the latter in non-Newtonian situations). In particular this applies to Quantum Mechanics, where neither the notion of a 'test particle' exists, nor does there exist an obvious worldline the equation of which could provide the sought-for link. So, for general states, it remains logically unclear what a possible equality of somehow defined inertial and gravitational masses implies for the universality of free fall, though it might make contextual sense for special states. However, that state dependency means that it cannot be used as a fundamental principle. 


\section{Conclusions}

In this paper we extended the calculation of [1] of a Hamiltonian describing an electromagnetically interacting two-particle system to non-flat spacetimes representing weak gravitational fields in the sense of being close to Minkowski spacetime. Starting from first principles we performed a post-Newtonian expansion in terms of the inverse velocity of light that led to leading-order corrections comprising special- and general-relativistic effects. The former were fully encoded in [1, but the latter are new. Our first principles were the special-relativistic action of two equally but oppositely charged particles with their electromagnetic interaction up to, and including, terms of order $c^{-2}$, the minimal coupling scheme for gravity, and canonical quantisation applied to the degrees of freedom in the Hamiltonian formalism. As in [1] we neglected all terms of third and higher order in $c^{-1}$, which physically means that we neglected radiation-reaction and also that we avoided obstructions on the applicability of the Hamiltonian formalism that result from the infamous 'no-interaction theorem' [28, whose impact only starts at the 6th order in a $c^{-1}$ expansion [29].

As for [1] in the gravity-free case, we see the virtue of our calculation in its firm rooting in explicitly spelled out principles, that leave no doubt concerning the questions of consistency and completeness of relativistic corrections. This, in our opinion, distinguishes our work from previous ones by other authors, who were also concerned with the coupling of composite particle quantum systems - like atoms or molecules - to external gravitational fields, who phrase their account of relativistic corrections in terms of semi-classical notions, like smooth worldlines and comparisons of their associated lengths (i.e. 'proper time' and 'redshift'); e.g. [3 8 ]. We emphasised in the introduction and also in our discussion of the Equivalence Principle that answers to the fundamental question of gravity-matter coupling in Quantum Mechanics should not be based on a priori restricted states that imply a semi-classical behaviour of some of the (factorising) degrees of freedom. Rather, they should apply to all states in an equally valid fashion.

Similar to the gravity-free case, we now derived the result that the centre of mass motion of the system can be viewed as that of a 'composite point particle', including in its mass the internal energy of the system. This result may be anticipated in a heuristic fashion on semi-classical grounds, but, as seen, its proper derivation requires some efforts. We stress once more that for this interpretation it was crucial to express the Hamiltonian in terms of the physical space-time metric. As a result, our work lends justification to current experimental proposals in atom interferometry that so far were based on these heuristic ideas on the basis of which completeness of the relativistic effects could not be reliably judged; e.g. [3 8 . Moreover, it also applies to new experimental setups, like that of an ion trap in a gravitational field, currently under investigation [30] in extension of [31]. Finally we mention that due to our explicit parametrisation of the gravitational field by means of the Eddington-Robertson parameters, our formulae will also apply to possible quantum tests of General Relativity against test theories within that class. 


\section{Acknowledgements}

We thank Klemens Hammerer for pointing out reference [1] and asking questions that initiated and encouraged the work reported here, as well as for critical reading of the manuscript and the suggestion of improvements. This work was supported by the Deutsche Forschungsgemeinschaft through the Collaborative Research Centre 1227 (DQmat), project B08. We thank all members for fruitful and encouraging discussions on topics related to the one dealt with in this paper.

\section{A. Formulae from the paper of Sonnleitner and Barnett}

In this appendix we reproduce all formulae from [1] that are used in the main text, while providing a little context. We use the original numbering, prepended with ' 1$]$.. For formulae containing an error in [1, we give here a corrected version; the corrections are highlighted in red and the number is marked with a star.

The classical Lagrangian for two particles interacting with electromagnetic potentials ${ }^{14}$ is

$$
\begin{aligned}
L= & -\sum_{i=1,2} m_{i} c^{2} \sqrt{1-\dot{\mathbf{r}}_{i}^{2} / c^{2}}+\int \mathrm{d}^{3} \mathbf{x}\left(\mathbf{j} \cdot \mathbf{A}_{\mathrm{tot}}-\rho \phi_{\mathrm{tot}}\right) \\
& +\frac{\varepsilon_{0}}{2} \int \mathrm{d}^{3} \mathbf{x}\left[\left(\partial_{t} \mathbf{A}_{\mathrm{tot}}+\boldsymbol{\nabla} \phi_{\mathrm{tot}}\right)^{2}-c^{2}\left(\boldsymbol{\nabla} \times \mathbf{A}_{\mathrm{tot}}\right)^{2}\right] .
\end{aligned}
$$

Note that here, in the notation of [1], $\phi_{\text {tot }}$ is the total electric potential, not to be confused with our gravitational potential $\phi$ from the main text.

One then splits the electromagnetic potentials into internal (generated by the particles) and external parts, employs the Coulomb gauge and solves the Maxwell equations for the internal part in lowest order (see (10.A1), [1].A3). Inserting the internal potential solutions and expanding the kinetic terms for the particles, one arrives at the postNewtonian Lagrangian

$$
\begin{aligned}
L\left(\mathbf{r}_{1}, \dot{\mathbf{r}}_{1}, \mathbf{r}_{2}, \dot{\mathbf{r}}_{2}, \mathbf{A}^{\perp}, \dot{\mathbf{A}}^{\perp}\right) \\
=L_{\text {Darwin }}\left(\mathbf{r}_{1}, \dot{\mathbf{r}}_{1}, \mathbf{r}_{2}, \dot{\mathbf{r}}_{2}\right)+\frac{\varepsilon_{0}}{2} \int \mathrm{d}^{3} \mathbf{x}\left[\left(\partial_{t} \mathbf{A}^{\perp}\right)^{2}\right. \\
\left.\quad-c^{2}\left(\boldsymbol{\nabla} \times \mathbf{A}^{\perp}\right)^{2}\right]+\int \mathrm{d}^{3} \mathbf{x} \mathbf{j} \cdot \mathbf{A}^{\perp}, \\
L_{\text {Darwin }}\left(\mathbf{r}_{1}, \dot{\mathbf{r}}_{1}, \mathbf{r}_{2}, \dot{\mathbf{r}}_{2}\right) \\
=\frac{m_{1} \dot{\mathbf{r}}_{1}^{2}}{2}+\frac{m_{1} \dot{\mathbf{r}}_{1}^{4}}{8 c^{2}}+\frac{m_{2} \dot{\mathbf{r}}_{2}^{2}}{2}+\frac{m_{2} \dot{\mathbf{r}}_{2}^{4}}{8 c^{2}} \\
\quad-\frac{1}{4 \pi \varepsilon_{0}} \frac{e_{1} e_{2}}{r}\left(1-\frac{\dot{\mathbf{r}}_{1} \cdot \dot{\mathbf{r}}_{2}}{2 c^{2}}\right)+\frac{e_{1} e_{2}}{4 \pi \varepsilon_{0}} \frac{\left(\dot{\mathbf{r}}_{1} \cdot \mathbf{r}\right)\left(\dot{\mathbf{r}}_{2} \cdot \mathbf{r}\right)}{2 r^{3} c^{2}},
\end{aligned}
$$

\footnotetext{
${ }^{14}$ In the absence of gravity, as this is the situation considered in [1].
} 
where $\mathbf{r}=\mathbf{r}_{1}-\mathbf{r}_{2}$ and $r=|\mathbf{r}|$.

The classical Hamiltonian obtained by Legendre transforming this Lagrangian is

$$
\begin{aligned}
H= & \frac{\overline{\mathbf{p}}_{1}^{2}}{2 m_{1}}-\frac{\overline{\mathbf{p}}_{1}^{4}}{8 m_{1}^{3} c^{2}}+\frac{\overline{\mathbf{p}}_{2}^{2}}{2 m_{2}}-\frac{\overline{\mathbf{p}}_{2}^{4}}{8 m_{2}^{3} c^{2}}+\frac{1}{4 \pi \varepsilon_{0}} \frac{e_{1} e_{2}}{r}\left(1-\frac{\overline{\mathbf{p}}_{1} \cdot \overline{\mathbf{p}}_{2}}{2 m_{1} m_{2} c^{2}}\right) \\
& -\frac{e_{1} e_{2}}{4 \pi \varepsilon_{0}} \frac{\left(\overline{\mathbf{p}}_{1} \cdot \mathbf{r}\right)\left(\overline{\mathbf{p}}_{2} \cdot \mathbf{r}\right)}{2 r^{3} c^{2} m_{1} m_{2}}+\frac{\varepsilon_{0}}{2} \int \mathrm{d}^{3} \mathbf{x}\left[\left(\boldsymbol{\Pi}^{\perp} / \varepsilon_{0}\right)^{2}+c^{2}\left(\boldsymbol{\nabla} \times \mathbf{A}^{\perp}\right)^{2}\right],
\end{aligned}
$$

where $\overline{\mathbf{p}}_{i}=\mathbf{p}_{i}-e_{i} \mathbf{A}^{\perp}\left(\mathbf{r}_{i}\right)(\star)$.

The PZW transformation operator is

$$
U=\mathrm{e}^{-\mathrm{i} \Lambda}=\exp \left[-\frac{\mathrm{i}}{\hbar} \int \mathrm{d}^{3} \mathbf{x} \boldsymbol{\mathcal { P }}(\mathbf{x}, t) \cdot \mathbf{A}^{\perp}(\mathbf{x}, t)\right],
$$

where $\mathcal{P}$ is the polarisation field

$$
\begin{aligned}
\mathcal{P}(\mathbf{x}, t)= & \sum_{i=1,2} e_{i}\left[\mathbf{r}_{i}(t)-\mathbf{R}(t)\right] \\
& \times \int_{0}^{1} \mathrm{~d} \lambda \delta\left\{\mathbf{x}-\mathbf{R}(t)-\lambda\left[\mathbf{r}_{i}(t)-\mathbf{R}(t)\right]\right\}
\end{aligned}
$$

The transformation amounts to the following change of canonical momenta:

$$
\begin{aligned}
\mathbf{p}_{i} & \rightarrow U \mathbf{p}_{i} U^{\dagger}=\mathbf{p}_{i}+\hbar \nabla_{\mathbf{r}_{i}} \Lambda, \\
\mathbf{\Pi}^{\perp}(\mathbf{x}) & \rightarrow \mathbf{\Pi}^{\perp}(\mathbf{x})+\mathcal{P}^{\perp}(\mathbf{x}) .
\end{aligned}
$$

In electric dipole approximation, i.e. expanding to first order in $\overline{\mathbf{r}}_{i}:=\mathbf{r}_{i}-\mathbf{R}$, and using $\sum_{j=1,2} e_{j}=0$, we find

$$
\begin{aligned}
\hbar \boldsymbol{\nabla}_{\mathbf{r}_{1,2}} \Lambda \simeq & e_{1,2}\left[\mathbf{A}^{\perp}(\mathbf{R})+\left(\overline{\mathbf{r}}_{1,2} \cdot \boldsymbol{\nabla}\right) \mathbf{A}^{\perp}(\mathbf{R})\right] \\
& +\frac{e_{1} \mathbf{r}_{1}+e_{2} \mathbf{r}_{2}}{2} \times\left[\boldsymbol{\nabla} \times \mathbf{A}^{\perp}(\mathbf{R})\right] .
\end{aligned}
$$

Thus, under the PZW transformation and the dipole approximation the momenta transform as $\mathbf{p}_{i}-e_{i} \mathbf{A}\left(\mathbf{r}_{i}\right) \rightarrow \mathbf{p}_{i}+\mathbf{d} \times \mathbf{B}(\mathbf{R}) / 2(\star)$, where $\mathbf{d}$ is the dipole moment.

Terms of the form

$$
\frac{\mathbf{p}_{i} \cdot[\mathbf{d} \times \mathbf{B}(\mathbf{R})]}{m_{i} m_{j} c^{2}} \propto \frac{\left|\mathbf{p}_{i}\right|}{m_{i} c} \frac{|\mathbf{d} \cdot \mathbf{E}(\mathbf{R})|}{m_{j} c^{2}}
$$

are neglected, since the atom-light interaction energy is assumed much smaller than the internal atomic energy, which is in turn much smaller than the rest energies of the 
particles. The multipolar Hamiltonian in electric dipole approximation is then

$$
\begin{aligned}
H_{[\text {mult }]} \simeq & \frac{\left[\mathbf{p}_{1}+\frac{1}{2} \mathbf{d} \times \mathbf{B}(\mathbf{R})\right]^{2}}{2 m_{1}}+\frac{\left[\mathbf{p}_{2}+\frac{1}{2} \mathbf{d} \times \mathbf{B}(\mathbf{R})\right]^{2}}{2 m_{2}} \\
& -\frac{e^{2}}{4 \pi \varepsilon_{0} r}+\frac{\varepsilon_{0}}{2} \int \mathrm{d}^{3} \mathbf{x}\left[\left(\mathbf{\Pi}^{\perp}+\mathcal{P}_{d}^{\perp}\right)^{2} / \varepsilon_{0}^{2}+c^{2} \mathbf{B}^{2}\right] \\
& -\frac{\mathbf{p}_{1}^{4}}{8 m_{1}^{3} c^{2}}-\frac{\mathbf{p}_{2}^{4}}{8 m_{2}^{3} c^{2}}+\frac{e^{2}}{16 \pi \varepsilon_{0} c^{2} m_{1} m_{2}} \\
& \times\left[\mathbf{p}_{1} \cdot \frac{1}{r} \mathbf{p}_{2}+\left(\mathbf{p}_{1} \cdot \mathbf{r}\right) \frac{1}{r^{3}}\left(\mathbf{r} \cdot \mathbf{p}_{2}\right)+(1 \leftrightarrow 2)\right],
\end{aligned}
$$

where $\mathcal{P}_{d}=+\mathbf{d} \delta(\mathbf{x}-\mathbf{R})(\star)$ is the polarisation in electric dipole approximation.

Expressed in Newtonian centre of mass coordinates, the Hamiltonian is as follows:

$$
\begin{aligned}
H_{[\mathrm{com}]}= & H_{\mathrm{C}}+H_{\mathrm{A}}+H_{\mathrm{AL}}+H_{\mathrm{L}}+H_{\mathrm{X}}, \\
H_{\mathrm{C}}= & \frac{\mathbf{P}^{2}}{2 M}\left[1-\frac{\mathbf{P}^{2}}{4 M^{2} c^{2}}-\frac{1}{M c^{2}}\left(\frac{\mathbf{p}_{\mathbf{r}}^{2}}{2 \mu}-\frac{e^{2}}{4 \pi \varepsilon_{0} r}\right)\right], \\
H_{\mathrm{A}}= & \frac{\mathbf{p}_{\mathbf{r}}^{2}}{2 \mu}\left(1-\frac{m_{1}^{3}+m_{2}^{3}}{M^{3}} \frac{\mathbf{p}_{\mathbf{r}}^{2}}{4 \mu^{2} c^{2}}\right)-\frac{e^{2}}{4 \pi \varepsilon_{0}} \\
& \times\left[\frac{1}{r}+\frac{1}{2 \mu M c^{2}}\left(\mathbf{p}_{\mathbf{r}} \cdot \frac{1}{r} \mathbf{p}_{\mathbf{r}}+\mathbf{p}_{\mathbf{r}} \cdot \mathbf{r} \frac{1}{r^{3}} \mathbf{r} \cdot \mathbf{p}_{\mathbf{r}}\right)\right], \\
H_{\mathrm{AL}}= & -\mathbf{d} \cdot \mathbf{E}^{\perp}(\mathbf{R})+\frac{1}{2 M}\{\mathbf{P} \cdot[\mathbf{d} \times \mathbf{B}(\mathbf{R})]+\text { H.c. }\} \\
& -\frac{m_{1}-m_{2}}{4 m_{1} m_{2}}\left\{\mathbf{p}_{\mathbf{r}} \cdot[\mathbf{d} \times \mathbf{B}(\mathbf{R})]+\mathrm{H} . \mathrm{c.}\right\} \\
& +\frac{1}{8 \mu}(\mathbf{d} \times \mathbf{B}(\mathbf{R}))^{2}+\frac{1}{2 \varepsilon_{0}} \int \mathrm{d}^{3} \mathbf{x} \mathcal{P}_{d}^{\perp^{2}}(\mathbf{x}, t), \\
H_{\mathrm{L}}= & \frac{\varepsilon_{0}}{2} \int \mathrm{d}^{3} \mathbf{x}\left(\mathbf{E}^{\perp^{2}}+c^{2} \mathbf{B}^{2}\right), \\
H_{\mathrm{X}}= & -\frac{\left(\mathbf{P} \cdot \mathbf{p}_{\mathbf{r}}\right)^{2}}{2 M^{2} \mu c^{2}}+\frac{e^{2}}{4 \pi \varepsilon_{0} r} \frac{(\mathbf{P} \cdot \mathbf{r} / r)^{2}}{2 M^{2} c^{2}} \\
& +\frac{m_{1}-m_{2}}{2 \mu M^{2} c^{2}}\left\{\left(\mathbf{P} \cdot \mathbf{p}_{\mathbf{r}}\right) \mathbf{p}_{\mathbf{r}}^{2} / \mu-\frac{e^{2}}{8 \pi \varepsilon_{0}}\right. \\
& \left.\times\left[\frac{1}{r} \mathbf{P} \cdot \mathbf{p}_{\mathbf{r}}+\frac{1}{r^{3}}(\mathbf{P} \cdot \mathbf{r})\left(\mathbf{r} \cdot \mathbf{p}_{\mathbf{r}}\right)+\text { H.c. }\right]\right\} .
\end{aligned}
$$

The canonical transformation into new coordinates $\mathbf{Q}, \mathbf{q}, \mathbf{p}$ used to eliminate the cross 
terms $H_{\mathrm{X}}$ reads as follows:

$$
\begin{aligned}
\mathbf{R}= & \mathbf{Q}+\frac{m_{1}-m_{2}}{2 M^{2} c^{2}}\left[\left(\frac{\mathbf{p}^{2}}{2 \mu} \mathbf{q}+\text { H.c. }\right)-\frac{e^{2}}{4 \pi \varepsilon_{0} q} \mathbf{q}\right] \\
& -\frac{1}{4 M^{2} c^{2}}[(\mathbf{q} \cdot \mathbf{P}) \mathbf{p}+(\mathbf{P} \cdot \mathbf{p}) \mathbf{q}+\text { H.c. }] \\
\mathbf{r}= & \mathbf{q}+\frac{m_{1}-m_{2}}{2 \mu M^{2} c^{2}}[(\mathbf{q} \cdot \mathbf{P}) \mathbf{p}+\text { H.c. }]-\frac{\mathbf{q} \cdot \mathbf{P}}{2 M^{2} c^{2}} \mathbf{P} \\
\mathbf{p}_{\mathbf{r}}= & \mathbf{p}+\frac{\mathbf{p} \cdot \mathbf{P}}{2 M^{2} c^{2}} \mathbf{P}-\frac{m_{1}-m_{2}}{2 M^{2} c^{2}} \\
& \times\left[\frac{\mathbf{p}^{2}}{\mu} \mathbf{P}-\frac{e^{2}}{4 \pi \varepsilon_{0}}\left(\frac{1}{q} \mathbf{P}-\frac{1}{q^{3}}(\mathbf{P} \cdot \mathbf{q}) \mathbf{q}\right)\right]
\end{aligned}
$$

The internal electromagnetic potentials up to our order of approximation (thus neglecting retardation) are as follows:

$$
\begin{aligned}
\phi_{\mathrm{el}, \mathrm{ng}}(\mathbf{x}, t)= & \frac{1}{4 \pi \varepsilon_{0}} \int \mathrm{d}^{3} \mathbf{x}^{\prime} \frac{\rho\left(\mathbf{x}^{\prime}, t\right)}{\left|\mathbf{x}-\mathbf{x}^{\prime}\right|} \\
\mathcal{A}_{\mathrm{ng}}^{\perp}(\mathbf{x}, t) \simeq & \frac{\mu_{0}}{4 \pi} \int \mathrm{d}^{3} \mathbf{x}^{\prime} \frac{\mathbf{j}\left(\mathbf{x}^{\prime}, t\right)}{\left|\mathbf{x}-\mathbf{x}^{\prime}\right|}+\frac{\mu_{0}}{(4 \pi)^{2}} \int \mathrm{d}^{3} \mathbf{x}^{\prime} \\
& \times \int \mathrm{d}^{3} \mathbf{x}^{\prime \prime} \frac{\mathbf{x}-\mathbf{x}^{\prime}}{\left|\mathbf{x}-\mathbf{x}^{\prime}\right|^{3}} \frac{\mathbf{j}\left(\mathbf{x}^{\prime \prime}, t\right) \cdot\left(\mathbf{x}^{\prime}-\mathbf{x}^{\prime \prime}\right)}{\left|\mathbf{x}^{\prime}-\mathbf{x}^{\prime \prime}\right|^{3}} \\
= & \frac{\mu_{0}}{8 \pi} \sum_{i=1,2} e_{i}\left\{\frac{\dot{\mathbf{r}}_{i}}{\left|\mathbf{x}-\mathbf{r}_{i}\right|}+\frac{\left(\mathbf{x}-\mathbf{r}_{i}\right)\left[\dot{\mathbf{r}}_{i} \cdot\left(\mathbf{x}-\mathbf{r}_{i}\right)\right]}{\left|\mathbf{x}-\mathbf{r}_{i}\right|^{3}}\right\}
\end{aligned}
$$

Here we have changed the variable names of the potentials to conform to our notation; in particular we added the suffix 'ng', standing for 'non-gravitational'. 


\section{References}

[1] M. Sonnleitner and S. M. Barnett, "Mass-energy and anomalous friction in quantum optics," Phys. Rev. A 98, 042106 (2018).

[2] S. Dimopoulos, P. W. Graham, J. M. Hogan, and M. A. Kasevich, "General relativistic effects in atom interferometry," Phys. Rev. D 78, 042003 (2008).

[3] M. Zych, F. Costa, I. Pikovski, and Č. Brukner, "Quantum interferometric visibility as a witness of general relativistic proper time," Nat. Commun. 2, 505 (2011).

[4] I. Pikovski, M. Zych, F. Costa, and Č. Brukner, "Universal decoherence due to gravitational time dilation," Nat. Phys. 11, 668-672 (2015).

[5] A. Roura, "Gravitational redshift in quantum-clock interferometry," (2018), arXiv:1810.06744 [physics.atom-ph] .

[6] E. Giese, A. Friedrich, F. Di Pumpo, A. Roura, W. P. Schleich, D. M. Greenberger, and E. M. Rasel, "Proper time in atom interferometers: Diffractive versus specular mirrors," Phys. Rev. A 99, 013627 (2019).

[7] S. Loriani, A. Friedrich, C. Ufrecht, F. Di Pumpo, S. Kleinert, S. Abend, N. Gaaloul, C. Meiners, C. Schubert, D. Tell, E. Wodey, M. Zych, W. Ertmer, A. Roura, D. Schlippert, W. P. Schleich, E. M. Rasel, and E. Giese, "Interference of clocks: A quantum twin paradox," Sci. Adv. 5, eaax8966 (2019).

[8] M. Zych, Ł. Rudnicki, and I. Pikovski, "Gravitational mass of composite systems," Phys. Rev. D 99, 104029 (2019).

[9] K.-P. Marzlin, "Dipole coupling of atoms and light in gravitational fields," Phys. Rev. A 51, 625-631 (1995)

[10] K. S. Thorne, D. L. Lee, and A. P. Lightman, "Foundations for a theory of gravitation theories," Phys. Rev. D 7, 3563-3578 (1973).

[11] M. Sonnleitner, N. Trautmann, and S. M. Barnett, "Will a decaying atom feel a friction force?" Phys. Rev. Lett. 118, 053601 (2017)

[12] S. M. Barnett and M. Sonnleitner, "Vacuum friction," J. Mod. Opt. 65, 706-712 (2018).

[13] C. G. Darwin, "The dynamical motions of charged particles," Philos. Mag. J. Sci. 39, 537-551 (1920)

[14] E. A. Power and S. Zienau, "Coulomb gauge in non-relativistic quantum electrodynamics and the shape of spectral lines," Philos. Trans. R. Soc. Lond. A 251, 427-454 (1959) 
[15] R. G. Woolley, "Molecular quantum electrodynamics," Proc. R. Soc. Lond. A 321, $557-572(1971)$

[16] M. Babiker and R. Loudon, "Derivation of the Power-Zienau-Woolley Hamiltonian in quantum electrodynamics by gauge transformation," Proc. R. Soc. Lond. A 385, 439-460 (1983).

[17] C. M. Will, Theory and Experiment in Gravitational Physics, revised ed. (Cambridge University Press, Cambridge, 1993) first edition 1981.

[18] C. Lämmerzahl, "A Hamilton operator for quantum optics in gravitational fields," Phys. Lett. A 203, 12-17 (1995).

[19] P. K. Schwartz and D. Giulini, "Post-Newtonian corrections to Schrödinger equations in gravitational fields," Class. Quantum Grav. 36, 095016 (2019).

[20] J. D. Jackson, Classical Electrodynamics, 3rd ed. (John Wiley \& Sons, New York, 1998).

[21] C. W. Misner, K. S. Thorne, and J. A. Wheeler, Gravitation (W. H. Freeman, San Francisco, 1973).

[22] S. W. Hawking and G. F. R. Ellis, The Large Scale Structure of Space-Time Cambridge Monographs on Mathematical Physics (Cambridge University Press, Cambridge, 1973).

[23] C. Anastopoulos and B. L. Hu, "Equivalence principle for quantum systems: dephasing and phase shift of free-falling particles," Class. Quantum Grav. 35, 035011 (2018).

[24] D. Puetzfeld, C. Lämmerzahl, and B. Schutz, Equations of Motion in Relativistic Gravity, Fundamental Theories of Physics, Vol. 179 (Springer, Cham, 2015).

[25] M. Visser, "Quantum mechanix plus Newtonian gravity violates the universality of free fall," Int. J. Mod. Phys. D 26, 1743027 (2017).

[26] M. Zych and Č. Brukner, "Quantum formulation of the Einstein equivalence principle," Nat. Phys. 14, 1027-1031 (2018).

[27] D. Giulini, "Equivalence principle, quantum mechanics, and atom-interferometric tests," in Quantum Field Theory and Gravity, edited by F. Finster, O. Müller, M. Nardmann, J. Tolksdorf, and E. Zeidler (Springer, Basel, 2012) Chap. 16, pp. 345-370, arXiv:1105.0749v2.

[28] D. G. Currie, T. F. Jordan, and E. C. G. Sudarshan, "Relativistic invariance and Hamiltonian theories of interacting particles," Rev. Mod. Phys. 35, 350-375 (1963).

[29] J. Martin and J. L. Sanz, "No-interaction theorem of Currie, Jordan and Sudarshan. Expansions in $c^{-1}$," J. Math. Phys. 19, 780-788 (1978). 
[30] V. Martínez et al., In preparation.

[31] R. Haustein, G. J. Milburn, and M. Zych, "Mass-energy equivalence in harmonically trapped particles," (2019), arXiv:1906.03980 [quant-ph]. 